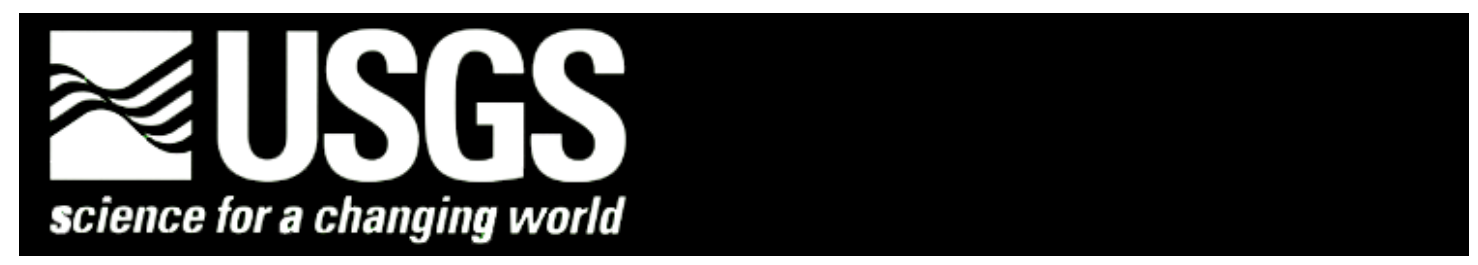

\title{
Comprehensive Areal Model of Earthquake-induced Landslides: Technical Specification and User Guide
}

\author{
By
}

Scott B. Miles and David K. Keefer

Open-File Report 2007-1072

2007

This report has not been reviewed for conformity with U.S. Geological Survey editorial standards or with the North American Stratigraphic code. Any use of trade, product, or firm names is for descriptive purposes only and does not imply endorsement by the U.S. Government.

\section{U.S. DEPARTMENT OF THE INTERIOR}

U.S. GEOLOGICAL SURVEY 


\section{Abstract}

This report describes the complete design of a comprehensive areal model of earthquakeinduced landslides (CAMEL). This report presents the design process, technical specification of CAMEL, and a guide to using the accompanying source code and template ESRI ArcGIS ${ }^{\mathrm{TM}}$ map document file for applying CAMEL. CAMEL is a regional-scale model of earthquake-induced landslide hazard developed using fuzzy logic systems. CAMEL currently estimates areal landslide concentration (number of landslides per square kilometer) of six aggregated types of earthquake-induced landslides - three types each for rock and soil. 
Table of Contents

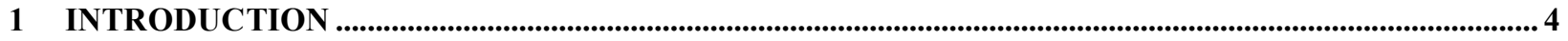

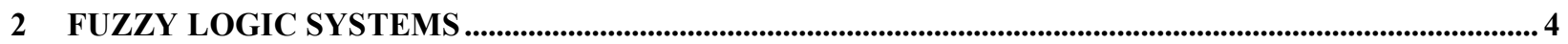

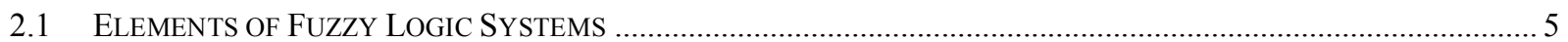

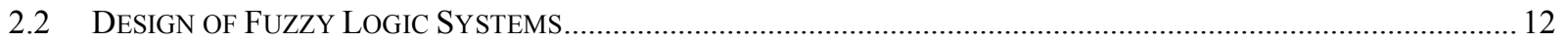

3 CAMEL KNOWLEDGE ELICITATION .............................................................................................................. 15

4 CAMEL DESIGN AND SPECIFICATION ........................................................................................................ 17

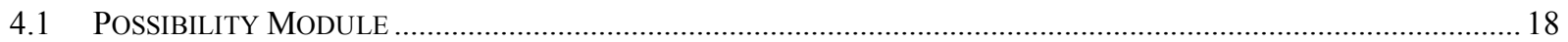

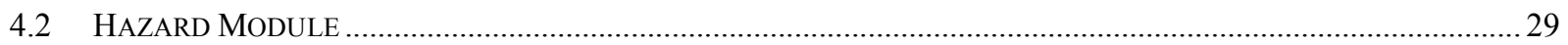

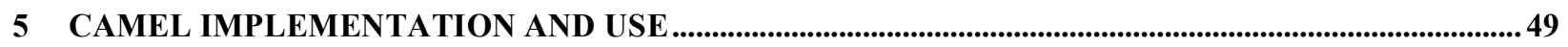

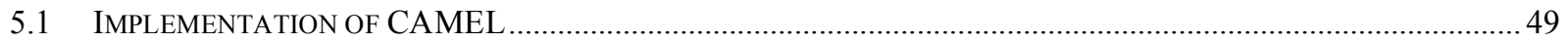

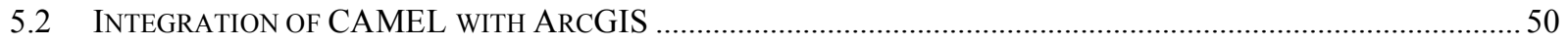

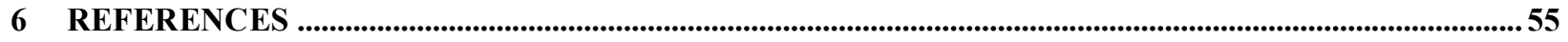

APPENDIX A: COMPLETE SPECIFICATION OF HAZARD MODULE RULE-BLOCKS FOR CAMEL

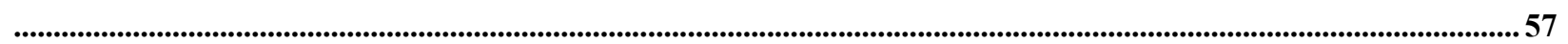

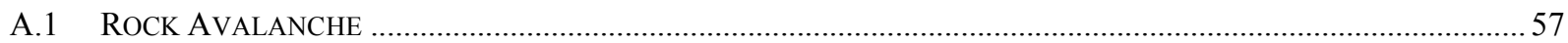

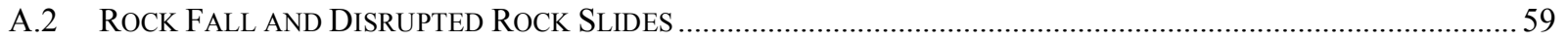

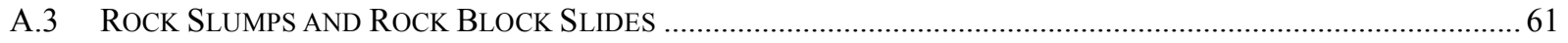

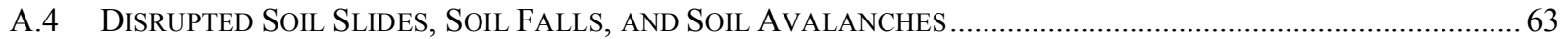

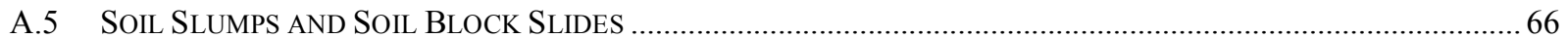

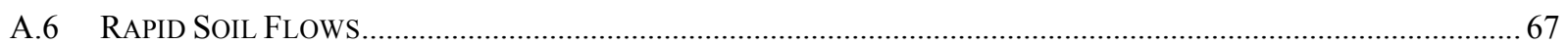




\section{Introduction}

This report describes the complete design of a comprehensive areal model of earthquakeinduced landslides (CAMEL). This report presents the design process, technical specification of CAMEL. It also provides a guide to using the CAMEL source code and template ESRI ArcGIS $^{\mathrm{TM}}$ map document file for applying CAMEL, both of which can be obtained by contacting the authors. CAMEL is a regional-scale model of earthquake-induced landslide hazard developed using fuzzy logic systems. CAMEL currently estimates areal landslide concentration (number of landslides per square kilometer) of six aggregated types of earthquake-induced landslides - three types each for rock and soil.

The report is presented in four sections. The first section describes the salient aspects of fuzzy logic systems to aid understanding of the CAMEL technical specification, presenting first general concepts and then a discussion of the fuzzy systems design process. The reader is referred to Berkan and Trubatch (1997) and Mendel (2001) for an in-depth discussion of fuzzy logic theory and design guidance, which not needed for use of CAMEL. The second section discusses the knowledge on which CAMEL was designed and how this knowledge was elicited. The third section specifies the structure, elements, and functionality of the fuzzy logic system that forms CAMEL, with a complete specification given as an appendix. The final section of this report describes the current implementation of CAMEL in ESRI's ArcGIS ${ }^{\mathrm{TM}}$ software. The section describes how to set up and use CAMEL with ESRI ArcMap ${ }^{\mathrm{TM}}$.

\section{Fuzzy Logic Systems}

Fuzzy logic systems are based on the concept of fuzzy sets. Fuzzy sets were introduced by Zadeh (1965) and are an infinite-valued extension of Boolean or "crisp" sets. Fuzzy sets represent the degree to which something is a member of a particular category, as opposed to the Boolean concept that category membership is represented as either completely true or false. Fuzzy logic systems are a series of interdependent blocks of IF-THEN rules, which relate input variables to a particular output variable. Based largely on Berkan and Trubatch (1997), the remainder of the section defines and illustrates the fundamental concepts of fuzzy logic systems 
and goes on to describe the general fuzzy logic systems design process.

\subsection{Elements of Fuzzy Logic Systems}

\subsubsection{Fuzzy Sets}

Fuzzy sets expand on the concept of crisp Boolean sets by representing the level of membership to a particular category - zero (0) indicating that membership is unambiguously false and one (1) indicating membership is unambiguously true. An example is useful to understand the differences between crisp and fuzzy sets. Take for example three sites: Site A is 23 kilometers from a fault zone, Site B is 27 kilometers away, and Site C is 75 kilometers away (Figure 1). Are these sites close to the seismic source? A crisp set requires definition of some function - typically a numerical range - to determine whether or not each site is a member of the category "close." Using this function, each site's distance value can be tested to determine whether membership is true (1) or false (0). If a range of 0 to 25 kilometers is chosen to crisply define "close", it is true (1) that Site A is a member of "close", while it is false (0) for Sites B and C. Pairing the site distances with the truth-value ( 0 or 1$)$ can represent this set: $23 \mathrm{~km} / 1.0$, $27 \mathrm{~km} / 0.0,75 \mathrm{~km} / 0.0)$. Sites A and B are only 4 kilometers apart, but A is classified as being close to the source and B is not.

With fuzzy sets, category membership is represented as a value between and including 0 and 1. This value is referred to as a truth value. The membership function in fuzzy logic then specifies not only where membership is true (1) and false (0), but how the truth value changes between 0 and 1 (y-axis) with respect to the function's domain (x-axis). Membership functions can have many different mathematical definitions, with the simplest and most commons ones being piece-wise linear functions. Membership functions have two fundamental requirements: (1) the function monotonically increases or decreases between 0 and 1 ; (2) the function has only one point (or region of points) corresponding to 1. For our example asking what sites are close to the seismic source, Figure 1 illustrates an arbitrary membership function defining the possible fuzzy sets for the category "close." The fuzzy set for this example then would be each distance value with the associated truth-value: $23 \mathrm{~km} / 0.6,27 \mathrm{~km} / 0.4,75 \mathrm{~km} / 0.0$. 


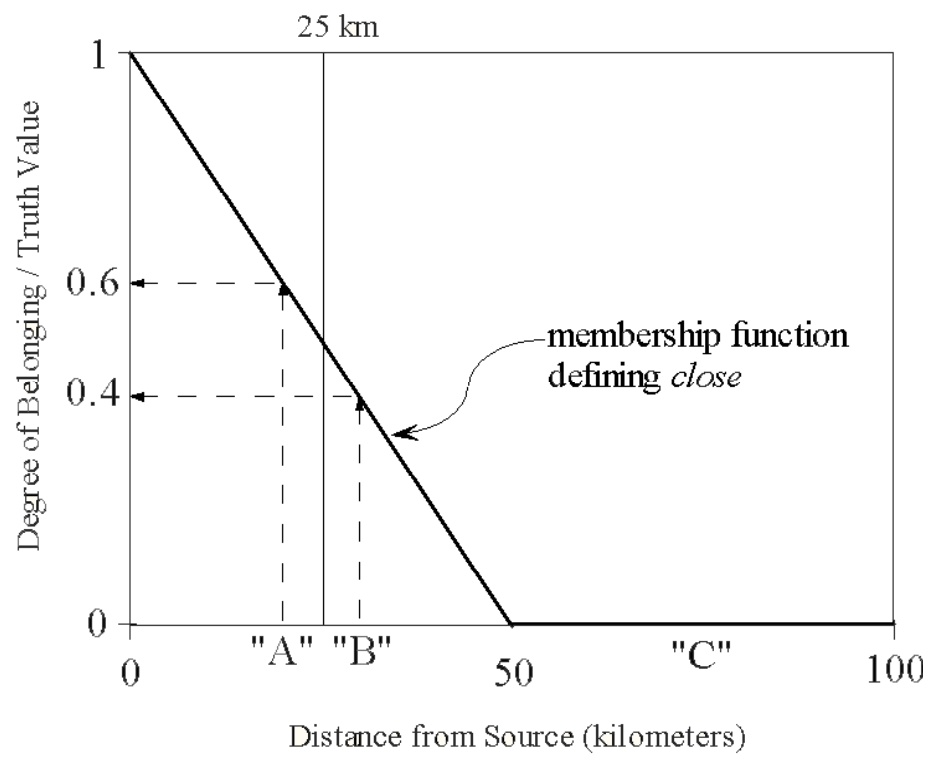

Figure 1. Example of crisp and fuzzy sets describing "close."

\subsubsection{Fuzzy Variables}

A variable in fuzzy logic systems is comprised of a series of membership functions defined with respect to a numeric domain. These membership functions are associated with the possible categories or "fuzzy values" of a particular variable. Each fuzzy value is typically assigned and referred to by a category label. Figure 2 shows an example of a variable Slope Angle, which is composed of the values "shallow," "moderate," and "steep" over the domain 0 to 50 degrees from the horizontal. For example, the statement "Slope Angle IS steep" indicates the variable Slope Angle has a value of "steep." Considering the membership function used to define "steep" shown in Figure 2, it is true that a slope having an angle from the horizontal of 35 degrees is "steep" to a degree of 0.7. That is, the truth value associated with the statement "Slope Angle IS steep" is 0.7 for a slope angle of 35 . 


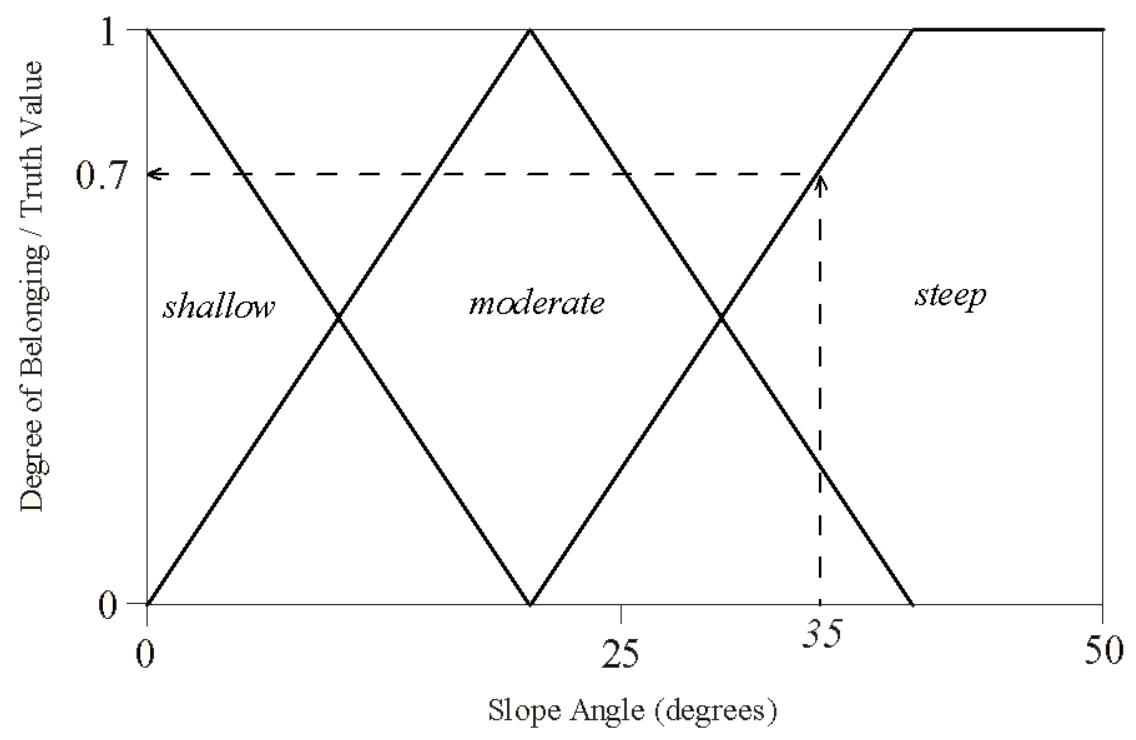

Figure 2. Example of the variable Slope having values of "shallow," "moderate," and "steep." The statement "Slope Angle IS steep" is associated with truth value of 0.7, if the slope angle is 35 degrees.

\subsubsection{Fuzzy Rules}

A rule within fuzzy logic systems is a computable IF-THEN statement relating variables. A simple IF-THEN rule can be constructed using one variable for the input and one variable for the output. An example of a rule is "IF Slope Angle IS steep THEN Landslide Hazard IS high" (Figure 3). The truth-value related to the input statement "Slope Angle IS steep" is basically assigned to the output statement "Landslide Hazard IS high." In the example of Figure 3, a slope value of 35 degrees results in a truth-value of 0.7 from the membership function for "steep," which is then passed on to the output variable value indicated in the IF-THEN rule; in this case "Landslide Hazard IS high." In other words, if "Slope Angle IS steep" with a truth value of 0.7, then the truth value for "Landslide Hazard IS high" is also 0.7. It is possible to assign a weight between 0 and 1 to any rule, which is multiplied to the output truth value. This weight is called the degree of support (DoS) weight. A DoS weight less than one reduces the influence of a particular rule. 


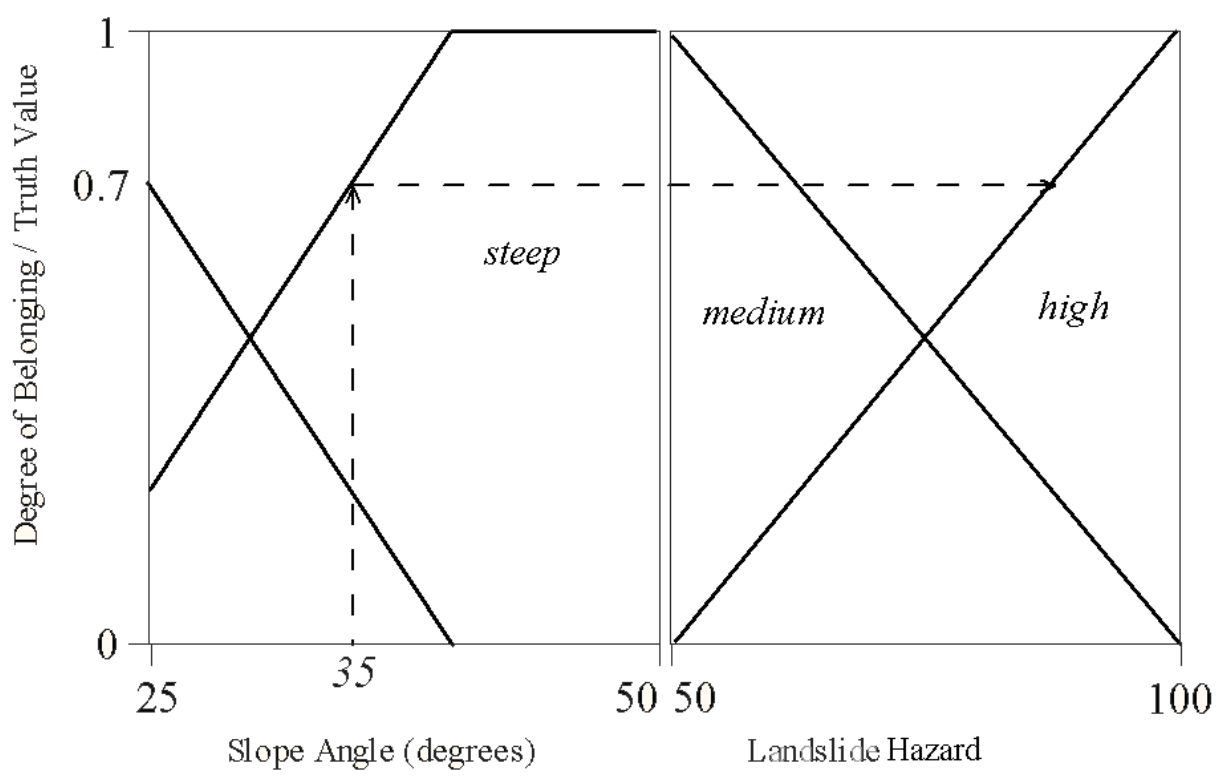

Figure 3. Example of passing on the input truth value to the output of the rule "IF Slope Angle IS steep THEN Landslide Hazard IS high."

\subsubsection{Combining Input Values for a Single IF-THEN Rule}

More complex IF-THEN rules can be constructed by combining multiple input variables using operators like AND and OR. These operators are conceptually the same as Boolean operators of the same name. The three most common fuzzy operators are AND (which passes on the lower truth-value associated with two statements), OR (which passes on the larger truthvalue associated with two statements), and PROD (which passes on the result of multiplying the truth-values associated with two statements). Consider a two-input rule, such as "IF Slope Angle IS steep AND Distance IS close THEN ...." Assuming the truth value associated with "Distance IS close" is 0.4 and the truth value for "Slope Angle IS steep" is 0.7, applying the AND operator results in a resultant truth value of 0.4 , which corresponds to the smaller of the two input truth-values. Combing the statements using the OR operator would result in a truthvalue of 0.7 - the larger of the input truth-values. Application of the PROD operator results in a combined truth-value of 0.28 - the product of the two input truth-values. Any number of fuzzy values can be combined using these operators.

\subsubsection{Combining Multiple Rules with the Same Output Values}

A fuzzy logic rule-block is a collection of fuzzy IF-THEN rules, with each rule mapping 
from any number of input variables to the same output variable. A complete rule-block will have a minimum of one rule for each output variable value (that are each associated with a membership function). Most rule-blocks, in fact, have more than one rule for each output variable value. In such cases, it is necessary to aggregate the multiple truth values determined from the multiple rules that map to the same output variable value. One aggregation operator that can be used is MAX, which takes the maximum truth-value of all the truth-values computed for a particular output variable value. Alternatively, the BSUM (for "bounded sum") aggregation operator can be used, which takes the bounded sum, that can't exceed 1.0, of all the truth values computed for a particular output fuzzy value. Table 1 lists an example rule-block containing four rules. There are two rules (rules 3 and 4) in which the output is "Landslide Hazard IS high." Consider if the output truth-value for rule 3 is 0.7 and is 0.5 for rule 4. Using MAX to aggregate the truth values for "Landslide Hazard IS high" would result in an aggregate truth value of 0.7 (the maximum of 0.5 and 0.7$)$. With BSUM, the sum of the two truth-values for "Landslide Hazard IS high" would exceed 1.0 and so would result in a aggregate truth-value of 1.0.

Table 1. A four-rule fuzzy rule-block relating Slope Angle and Distance to Landslide Hazard.

1. IF Slope Angle IS shallow THEN Landslide Hazard IS low

2. IF Slope Angle IS moderate AND Distance IS close THEN Landslide Hazard IS medium

3. IF Slope Angle IS steep THEN Landslide Hazard IS high

4. IF Lithology IS landslide deposit THEN Landslide Hazard IS high

\subsubsection{Combining Rules with Different Output Values}

To determine the overall result of a rule-block, all the contained rules are computed based on specific input data. The combined truth-values associated with each output value can be directly interpreted. For example, output truth-values for the rule-block of Table 1 might be determined as: "Landslide Hazard IS low" equal to 0; "Landslide Hazard IS medium" equal to 0.3; "Landslide Hazard IS high" equal to 1.0. The interpretation of this result would be that landslide hazard is unambiguously not low, but is partially medium and partially high, with evidence from input data indicating that the landslide hazard is significantly more high than medium. 
Alternatively, the truth-values for each of the output variable values can be defuzzified, meaning that a scalar value is determined within the output variable domain. There are several defuzzification methods. For modeling applications, the most common method is called the Center of Maximum (CoM) method. The CoM method takes a weighted average of the output variable domain values, using as weights the truth value associated with the center of each output variable membership function. Consider the example where the Landslide Hazard membership function maxima for "low," "medium," and "high" are associated with domain values of 0, 50, and 100 , respectively. Using truth values assumed in the examples above (low/0.0, medium/0.3, high/1.0 ), the defuzzified value using the CoM method would be:

$$
\frac{0 \cdot 0.0+50 \cdot 0.3+100 \cdot 1.0}{0.0+0.3+1.0}=88.5
$$

\subsubsection{Example Computation of a Simple Fuzzy System}

Presenting a complete graphical example for a simple fuzzy IF-THEN rule-block can summarize the elements of fuzzy logic systems. Figure 4 presents the fuzzy rule-block of Table 1, showing the membership functions for each variable as assumed above. Consider a case where the input data into the fuzzy logic system is Slope $=35$ degrees, Distance $=27 \mathrm{~km}$, Lithology is landslide deposit with a truth value of 0.5. Using BSUM to aggregate the same output values and CoM for defuzzification, a final crisp landslide hazard value of 88.5 is computed. 


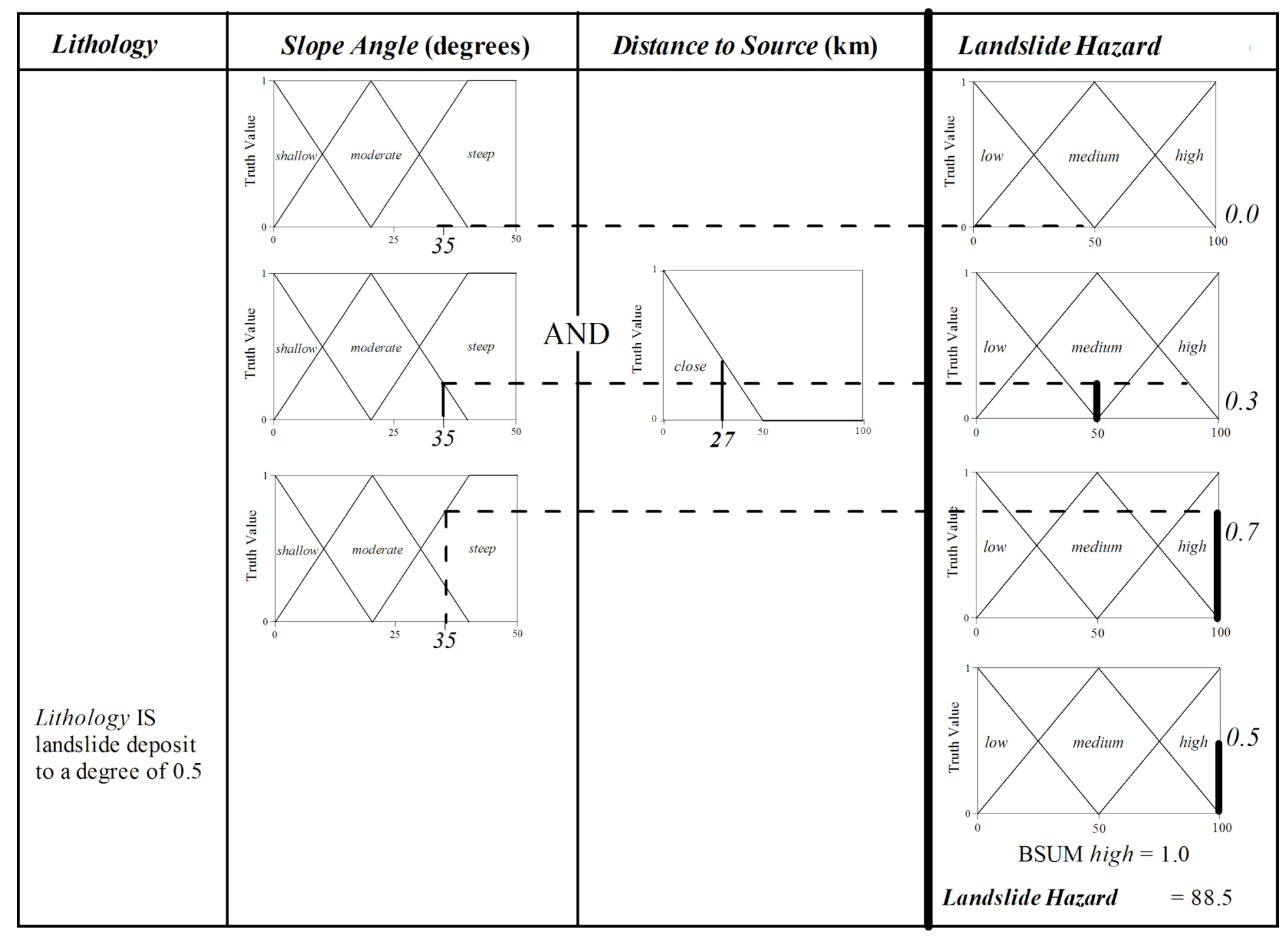

Figure 4. Complete example of a 4-variable, 4-rule fuzzy system (Table 1) for an input data scenario of Slope $=35$ degrees, Distance $=27 \mathrm{~km}$, and Lithology IS landslide deposit with a truth value of 0.5 . 


\subsection{Design of Fuzzy Logic Systems}

Assembling the elements of fuzzy logic systems, described above, to express a solution of a problem requires a significant design effort. The general design phases for developing CAMEL were based on the design guide of Berkan and Trubatch (1997). The basic design phases consist of (1) knowledge elicitation, (2) system design, and (3) implementation.

\subsubsection{Knowledge Elicitation}

Knowledge elicitation is the process of extracting useful and reproducible knowledge from experts, literature sources, quantitative data, and other models. Knowledge elicitation from experts requires direct observation of experts (e.g., a doctor making a diagnosis), face-to-face interviews, or the use of questionnaires. To extract knowledge from scientific literature, the text can be analyzed to identify the variables involved and their respective categories, as well as to pull out logical propositions. One sentence may yield several rules or several sentences might lead to a single rule. Descriptions of consequences or actions can be used to identify output variables, while inputs are often indicated by the descriptions of causes. Within scientific literature, much of the knowledge might be represented in graphical form, which might be possible to extract rules in a similar way as with empirical data. Otherwise, scientific charts and figures can be used to identify outputs, inputs, and their association. Direct translation of text can be difficult, if not impossible. Knowledge elicitation from empirical data involves extracting IF-THEN rules from a data set, either manually or automatically with the assistance of appropriate methods (e.g., artificial neural networks). Mathematical equations can be plotted and used to identify rules. The essence of fuzzy rule extraction is to identify clusters of data points that indicate an association with desired categories and determine the relationships between the categories of each variable. Conceptually, this process is similar to fitting a line to a scatterplot of data.

\subsubsection{System Design}

The model design phase serves to formalize the results of knowledge elicitation through various design tasks. Typically these tasks consist of (1) design of variables, (2) rule composition, and (3) and selecting the numerical method for translating inputs to ouputs (rule 
implication). These tasks must be performed in sequence, but the design process of course requires iteration.

\subsubsection{Variable Definition}

The first task for the design phase is the definition of variables. There are two general approaches to variable design: data-driven design and semantic design. The two approaches are opposite means of designing the same elements. The three elements for each variable in a fuzzy logic system include (1) the numeric domain in which the variable is valid, (2) membership functions, and (3) linguistic labels for each membership function. The primary goal of the variable design process is to determine the number of membership functions and their location in the domain. The difference between the data-driven and semantic design approaches is in the specification of membership functions. Data-driven design utilizes data that represents the desired system behavior or process. Semantic design uses heuristics or expert judgment. Semantic design is necessary when there is a lack of appropriate quantitative data to meet specific design criteria. Thus, the number of membership functions might correspond with the number of data clusters discovered or the number of heuristics that can be articulated for each variable. As with all design problems, iterations are often required and so data-driven and semantic design are not mutually exclusive in practice.

With the number and location specified, the shape of each membership function needs to be determined. In most cases, simple piece-wise linear functions (e.g., triangular or trapezoidal) are sufficient. Other shapes may be used to meet certain design criteria (e.g., how quickly or slowly the model transitions from one state to another) or to subsequently fine-tune the model, often with automated learning methods. Semantically appropriate labels should be selected for each membership function. Label selection is one of the essential elements for making fuzzy logic rules comprehendible.

\subsubsection{Rule Composition}

The next task is rule composition. In composing IF-THEN rules, individual statements (e.g., "Slope Angle IS steep") are combined using operators like AND and OR. A commonly used operator related to AND is the PROD operator, which is formulated as the product of two 
truth-values. Higher input truth values are required for PROD to yield the same output truth value as computed using AND. In practice, the OR operator may not be necessary because a single two-input rule using the OR operator is equivalent to two one-input rules when using CoM defuzzification (Li, 2002). Avoiding use of the OR operator or, more strictly, using singleinput rules has three critical advantages. First, the task of specifying rules for every input combination is avoided (Combs and Andrews, 1998). Second, computational performance of the system is improved (Lazzerini, 2000). Third, the resulting rule-block is easier to read and potentially understand (Guillaume, 2001).

After determining what fuzzy operator to employ to relate input variables, rule composition is completed by determining an appropriate strategy for organizing the rules. Examples of strategies include the following: competitive rules, cooperative rules, weighted rules, prioritized rules, and hierarchical rules. Each strategy (or some combination thereof) can be used in modeling a process; the choice of strategy depends on the particular design objectives (e.g., performance with missing input data). Competitive rules are rules that consider the same input variables (though likely different fuzzy values) to represent the same output variable. Cooperative rules, conversely, make use of different variables to represent the same output variable. Weighted rules express varying confidence or importance across some set of rules, using degree of support (DoS) weights. Rule prioritization refers to intentional sequencing of computing rules. The most common means of rule prioritization is the creation of a series of rule-blocks in which outputs from one rule-block are used as input in another.

\subsubsection{Rule Implication}

The final task of design is rule implication. Implication refers to the design elements that compute outputs from inputs statements. Rule implication design requires two decisions. A choice must be made regarding the means of combining different truth-values determined for the same output fuzzy values using either MAX or BSUM. MAX uses only the results of a single rule, while BSUM takes a bounded sum, not to exceed 1, of all rule truth-values. In contrast to MAX, BSUM potentially takes into account multiple rules. As a result, BSUM will always yield an equal or higher combined truth-value than MAX. Finally, the choice must be made 
regarding whether to defuzzify results and, if so, how.

\section{CAMEL Knowledge Elicitation}

The first step in the development of CAMEL was knowledge elicitation, which forms the basis for variable definition and composition of IF-THEN rules. For initial development, two sources of knowledge were used: scientific literature and expert judgment. This knowledge can be revised or supplemented in the future using empirical data, outputs from alternative models, or knowledge elicited from experts. Knowledge elicitation was done by compiling literature describing analyses of past landslide-triggering earthquakes and extracting relevant numerical or correlative information. Expert judgment was used to fill any gaps in knowledge from the literature. The primary literature sources used for knowledge elicitation included Keefer (2002), Liao et al. (2002), Hancox et al. (2002), Parise and Jibson, (2000), Bommer and Rodriguez (2000), Keefer (2000), Keefer (1999), Rodriguez et al., (1999), Keefer (1993), and Keefer (1984).

At this point in development, empirical data was not used as a source for knowledge elicitation, for three reasons. First, in general there are few landslide inventories (and fewer spatial databases) from historical earthquakes. Second, many of the available inventories do not provide detailed information, for example, with respect to different types of landslides. Lastly, for initial development of CAMEL it is useful to be able to use empirical data to help evaluate the quality of the foundational sources of knowledge. Mathematical equations, such as the many available equations for calculating Newmark displacement, were not used for initial development for two reasons. There do not exist mathematical models for each category and type of earthquake-induced landslides. Most critically, use of existing equations would constrain the choice of input and output variables. Further, few mathematical relationships exist that predict landslide concentration, especially for particular types of landslides. With the conceptual and algorithmic framework of CAMEL developed, empirical data can be used in the future to refine the variable definitions, individual rules or rule blocks, and rule weights.

The first step in the knowledge elicitation process was determination of input and output variables. The output variables were largely determined by the design objectives for CAMEL (i.e., prediction of landslide concentration for every landslide type). For initial development, we 
decided to reduce the number of output variables by aggregating similar landslide types based on the classification of Keefer (1984). Thus, ten distinct types are represented by the six output variables listed in Table 2. For this stage in the research, soil lateral spreads are not considered because of the significantly different type of data and knowledge needed to characterize this landslide type. An initial list of input variables was obtained by analyzing a cross-section of the landslide and earthquake ground-motion literature using text indexing and search software to help identify commonly sited indicators, conditioning factors, and independent variables. The potential list of input variables was pared down based on the specificity of information within the literature for each potential variable and by combining similar concepts into single variables. The input variables of CAMEL are specified and discussed later in the report.

Table 2. Aggregated types of earthquake-induced landslides, listed by category, modeled by CAMEL. Taxonomy and terminology after Keefer (1984).

\section{- Category I}

Disrupted rock falls and slides

Rock avalanches

Disrupted soil slides, falls, and avalanches

- Category II

Rock slumps and block slides

Soil slumps and block slides

- Category III*

Rapid soil flows

*Soil lateral spreads not considered

Examination of the summative literature revealed that there are two general types of knowledge with respect to past occurrence of earthquake-induced landslides. The first type of knowledge describes indicators or geologic conditions that suggest whether the occurrence of a particular type of landslide is possible. The second type of knowledge describes the variation in intensity of hazard with the increasing magnitude of particular variables. These two types of knowledge - indicators and intensifiers — serve as the organizing principle for the design of CAMEL. The specific knowledge and its role in the design of CAMEL are discussed in the following section specifying the fuzzy system elements of CAMEL. 


\section{CAMEL Design and Specification}

CAMEL is comprised of two modules, each made up of several IF-THEN rule-blocks to respectively determine the possibility and areal concentration of earthquake-induced landslides (Figure 5). The possibility module determines whether the occurrence of each respective landslide type is possible. The hazard module determines the relative hazard, expressed in units of landslides per square kilometer, for each possible landslide type. This modular framework serves to separate the implementation of the knowledge about possibility of occurrence from knowledge about relative hazard for each landslide type listed in Table 2.

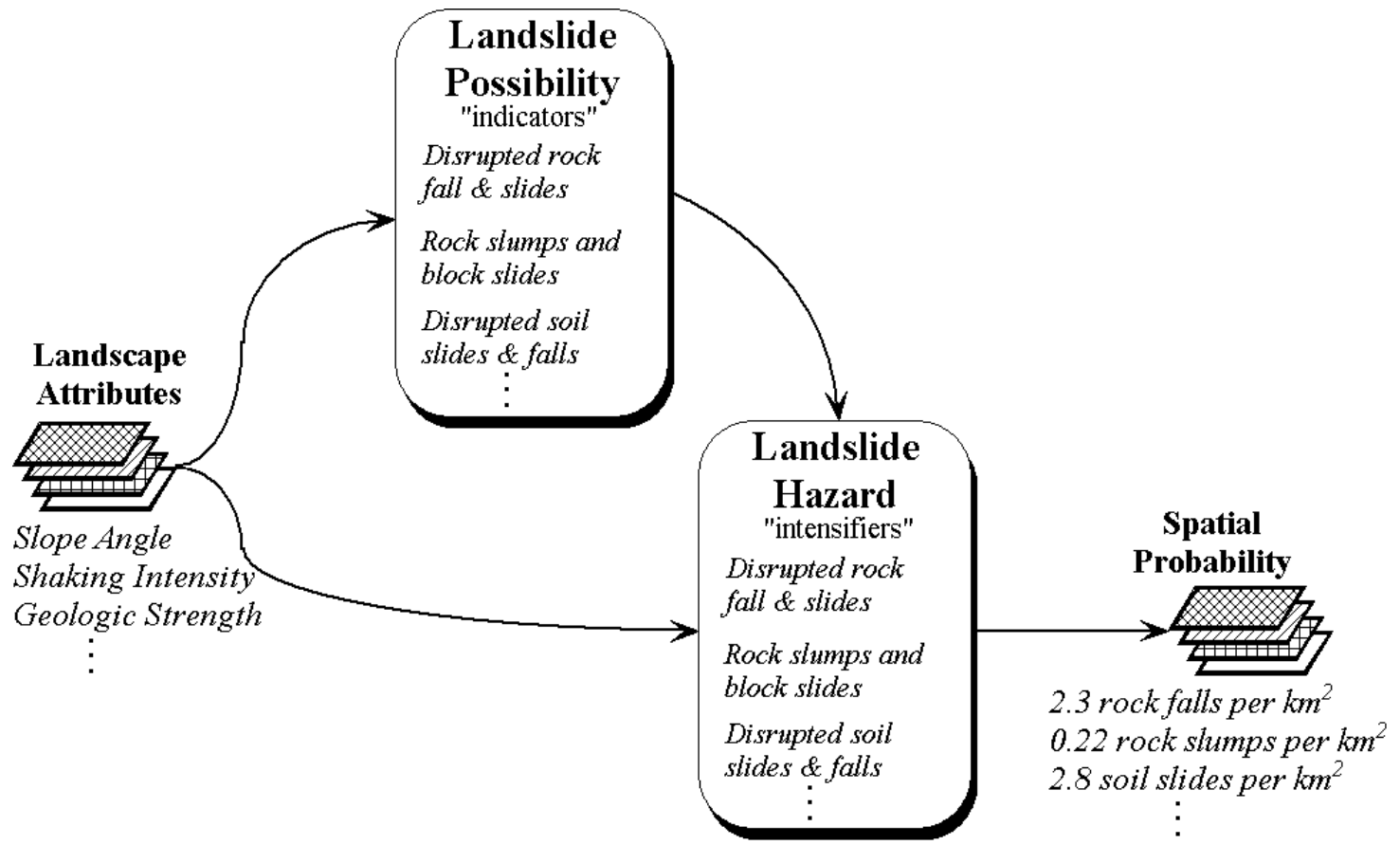

Figure 5. Two-module framework of CAMEL, including the possibility and hazard modules.

The two modules of CAMEL are described in detail below. The conceptual definition of each variable is described, in addition to the sources of knowledge. The input variables are defined graphically by presenting each variables set of membership functions, specified across a particular domain. Output variable definitions are presented in table form. The composition of each respective block of IF-THEN rules is described. Design choices that were made in translating elicited knowledge to the elements of CAMEL are discussed. Finally, any limitations of the particular design element are presented. 


\subsection{Possibility Module}

The structure and data flow of the possibility module is given in Figure 6. The module consists of seven input variables, together with seven corresponding rule blocks that are organized in series - a competitive arrangement. Each rule block contains rules associated with a specific variable to collectively determine the degree to which each landslide type is possible. If one indicator variable specifies that a particular landslide type is not possible, the overall result of this chain of rule-blocks will be that the particular landslide type is impossible. If all indicator variables show that a landslide type is possible, the minimum truth-value across these rule blocks is the output truth-value for the possibility module. The magnitude of the output truth-value indicates the degree to which the user-supplied input data suggests that each landslide type is possible based on the knowledge represented by the IF-THEN rules of the module. The AND operator is used for all rule blocks in the possibility module. PROD would result in lower computed possibilities because truth-values are combined through multiplication. This design choice means that CAMEL assumes landslide types are possible in the face of uncertainty. Lastly, all rules in the possibility module have an equal DoS weight of 1.0.

The possibility of each landslide type is expressed through the output variable LandslideType. LandslideType is a nominal variable (i.e., a crisp value cannot be determined through defuzzification) comprised of six categories corresponding to each landslide type modeled by CAMEL. Outputs associated with this variable are a fuzzy set with a truth-value computed for each of the six landslide types. If a truth-value is zero (0) for a particular landslide type, that type is considered to be impossible based on the inputs provided to CAMEL. Positive possibility values then indicate that the particular landslide type is possible to the degree indicated. 


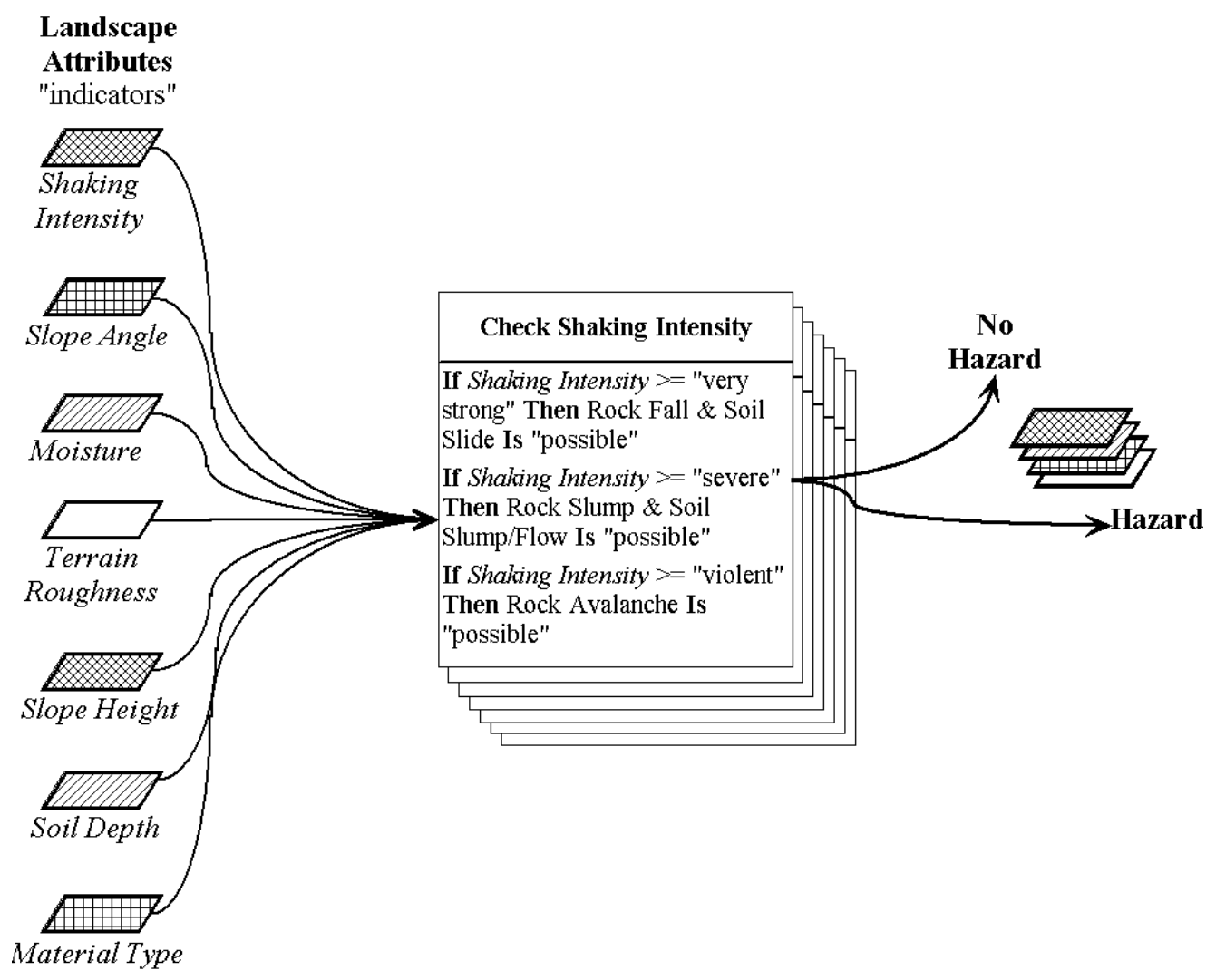

Figure 6. Possibility module of CAMEL.

Table 3 enumerates the indicator variables that comprise the inputs of the possibility module of CAMEL. The variables are listed in the sequence in which CAMEL considers each indicator. The specific sequence is arbitrary. Each indicator is briefly defined with the quantitative units, the domain, and the number and label of the fuzzy values for each variable. Variables that have a domain defined by a minimum value of -1 have an actual minimum of 0 . The negative value is a design technique to track whether the user has not provided an input value. For these variables, "missing" (data) is a valid data value (i.e., data does not have to be specified for the particular CAMEL variable to compute outputs). The design and specification of each indicator variable and associated rule block is described in detail below in the order listed in Table 3. 
Table 3. CAMEL Possibility Module Input Variables.

\begin{tabular}{|l|c|c|c|l|}
\hline Variable Name & Units & Min & Max & Fuzzy Value Labels \\
\hline pTerrainRough & $\begin{array}{c}\text { Slope of slope } \\
\text { angle }\end{array}$ & 0 & 40 & $\begin{array}{l}\text { planar } \\
\text { rough }\end{array}$ \\
\hline ShakeIntensity & $\begin{array}{c}\text { Shakemap } \\
\text { intensity } \\
\text { (MMI) }\end{array}$ & -1 & 12 & $\begin{array}{l}\text { missing } \\
\text { greater_than_7 } \\
\text { greater_than_8 } \\
\text { greater_than_9 }\end{array}$ \\
\hline pSoilDepth & Meters & -1 & 10 & $\begin{array}{l}\text { missing } \\
\text { shallow } \\
\text { deep }\end{array}$ \\
\hline pSlopeHeight & Meters & -1 & 300 & $\begin{array}{l}\text { missing } \\
\text { low } \\
\text { high }\end{array}$ \\
\hline pMaterialType & (linguistic) & - & - & $\begin{array}{l}\text { missing } \\
\text { rock } \\
\text { soil }\end{array}$ \\
\hline pMoisture & Percent & 0 & 100 & $\begin{array}{l}\text { more_than_moist } \\
\text { about_saturated }\end{array}$ \\
\hline pSlopeAngle & $\begin{array}{l}\text { Degrees from } \\
\text { the horizontal }\end{array}$ & 0 & 90 & $\begin{array}{l}\text { between_5and40 } \\
\text { between_15and40 } \\
\text { greater_than5 } \\
\text { greater_than_15 } \\
\text { greater_than_25 } \\
\text { greater_than_35 }\end{array}$ \\
\hline
\end{tabular}

\subsection{1 pTerrainRough}

Quantitative knowledge about slope curvature and earthquake-induced landslide hazard is relatively sparse. A study by Liao et al. (2002) found that a variable they referred to as "terrain roughness" correlated exceptionally well with the occurrence of landslides from the 1999 Chi-Chi, Taiwan earthquake. Landslides triggered during this event were primarily disrupted falls and slides. Terrain roughness is simply the unsigned slope curvature in profile. In other words it is the second derivative of elevation in the down-slope direction, with all calculation done in degrees. The two fuzzy values for pTerrainRough — "planar" and "rough"—lie on either side of a second-derivative value of 15 (Figure 7). Liao et al. (2002) found a significant increase in landslide concentration above this value. Liao et al. (2002) observed that there is some small possibility of having a landslide at terrain roughness values of as low as 5. An s-shape membership function is used rather than a straight-line curve because it results in very low truthvalues near a pTerrainRough of zero. No accommodation for missing data is made for 
pTerrainRough because slope curvature can be calculated from elevation data, which is likely to be used to calculate regional slope angle values (a requirement for running CAMEL).

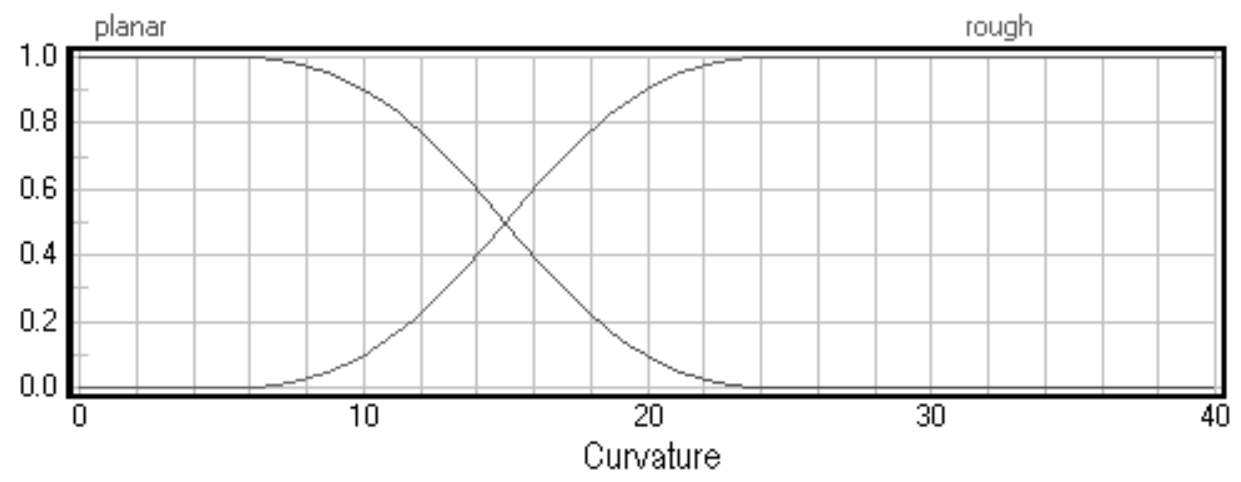

Figure 7. Membership functions for pTerrainRough.

The rules that determine possibility based on pTerrainRough are listed in Table 4. For Category I landslides, possibility is true only if pTerrainRough is "rough." It is assumed that terrain roughness is not a significant indicator for the remaining landslide types of CAMEL, and so possibility is true for either fuzzy value. This rule block is currently first in the sequence of the possibility module. Therefore, it has to consider all input combinations. Even though it is not important whether, for example, soil slumps have a "rough" pTerrainRough, this fuzzy value must be checked for.

Table 4. Possibility module rule block expressing knowledge about pTerrainRough.

\begin{tabular}{|l|l|l|}
\hline \multicolumn{1}{|c|}{ IF } & \multicolumn{2}{|c|}{ THEN } \\
\hline pTerrainRough & DoS & LandslideType7 \\
\hline rough & 1.00 & rock_fall \\
\hline rough & 1.00 & rock_avalanche \\
\hline rough & 1.00 & soil_slide \\
\hline rough & 1.00 & rock_slump \\
\hline rough & 1.00 & soil_slump \\
\hline rough & 1.00 & soil_flow \\
\hline planar & 1.00 & soil_slide \\
\hline planar & 1.00 & rock_slump \\
\hline planar & 1.00 & soil_slump \\
\hline planar & 1.00 & soil_flow \\
\hline
\end{tabular}




\subsection{2 pShakelntensity}

The minimum required shaking intensity required for each respective type of landslide is represented by the indicator pShakeIntensity. The units of the variable are based on the ShakeMap intensity scale (Wald et al., 1999), which is similar to the Modified Mercalli Intensity (MMI) scale, but is a ratio scale. This facilitates use of ShakeMap outputs, which are available in GIS data formats from actual or scenario events (http://earthquake.usgs.gov/shakemap/). As illustrated in Figure 8, pShakeIntensity is made up of three fuzzy values: "missing", “greater_than_7”, "greater_than_8”, and "greater_than_9." The lowest value is applied to Category I landslides, not including rock avalanches. The middle value is applied to Category II (coherent slides) and Category III landslides (rapid soil flows). The highest value is related to the occurrence of rock avalanches. MMI threshold values for rock avalanche were determined from minimum magnitude and distance information in Hancox et al. (2002) and Keefer (1984), which were then converted to MMI. The other values are based on Rodriguez et al. (1999) and Keefer (1984) (Figure 9). Each membership function increases from a truth-value of zero (0) at the lowest recorded minimum MMI threshold to a truth of one (1) at the maximum-recorded minimum MMI value. If no intensity data is provided, CAMEL will compute static susceptibility for each landslide type.

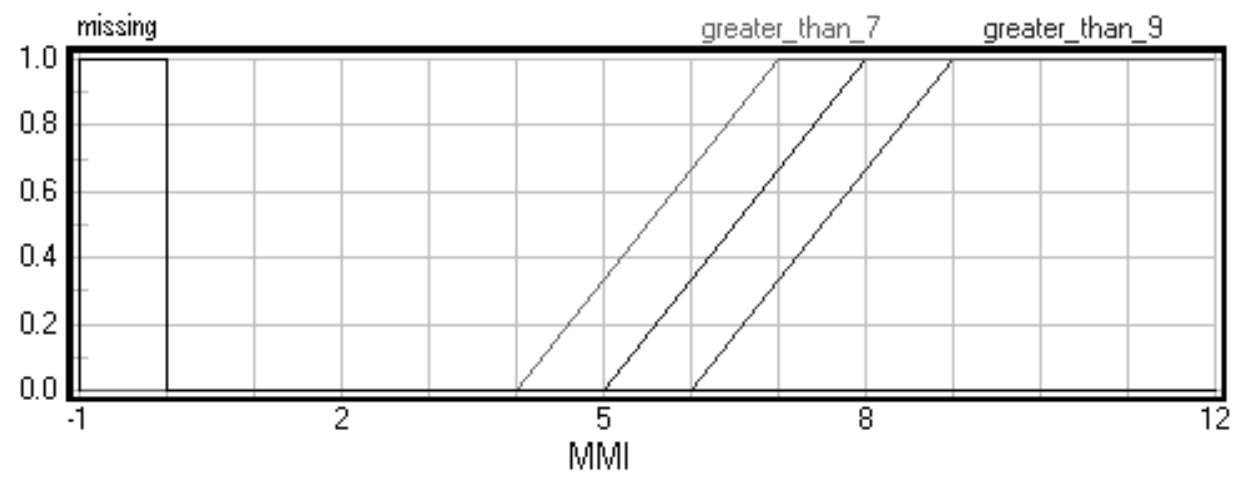

Figure 8. Membership functions for pShakeIntensity. 


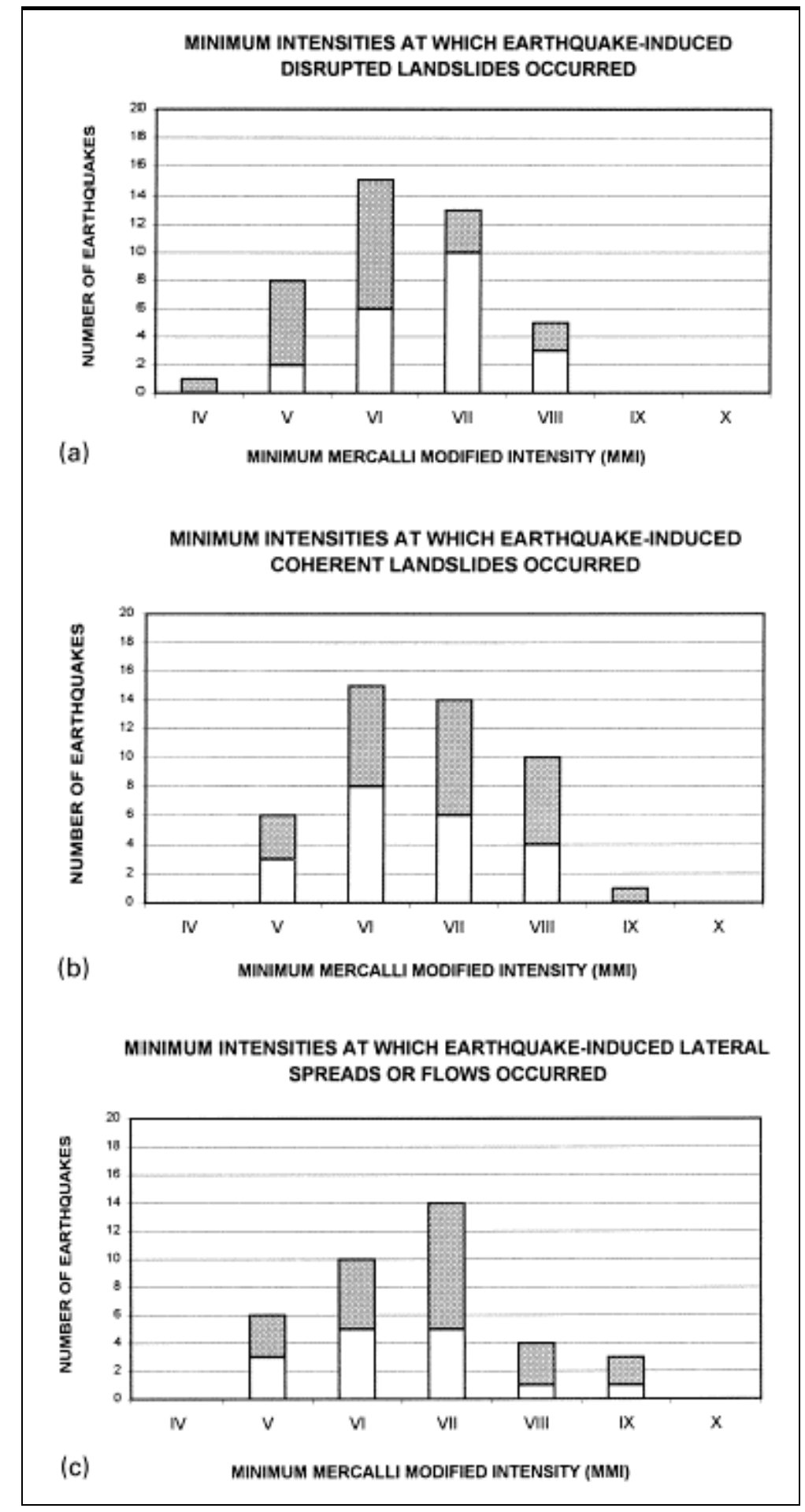

Figure 9. Minimum shaking intensity required for occurrence of different categories of landslides (Rodriguez et al., 1999). Gray boxes from Keefer (1984); white boxes from Rodgriguez et al. (1999).

The rules to determine possibility for each landslide type based on pShakeIntensity are 
listed in Table 5. LandslideType 7 is used to link this rule block with the previous rule block. As described above, AND is used to pass on the truth-value from the previous rule block. The last six rules of the rule block handle cases of missing data (to conduct static susceptibility analysis).

Table 5. Possibility module rule block expressing knowledge about pShakeIntensity.

\begin{tabular}{|l|l|l|l|}
\hline \multicolumn{2}{|c|}{ IF } & \multicolumn{2}{c|}{ THEN } \\
\hline LandslideType7 & pShakeIntensity & DoS & LandslideType6 \\
\hline \hline rock_fall & greater_than_7 & 1.00 & rock_fall \\
\hline soil_slide & greater_than_7 & 1.00 & soil_slide \\
\hline rock_slump & greater_than_8 & 1.00 & rock_slump \\
\hline soil_slump & greater_than_8 & 1.00 & soil_slump \\
\hline soil_flow & greater_than_8 & 1.00 & soil_flow \\
\hline rock_avalanche & greater_than_9 & 1.00 & rock_avalanche \\
\hline rock_fall & missing & 1.00 & rock_fall \\
\hline rock_avalanche & missing & 1.00 & rock_avalanche \\
\hline soil_slide & missing & 1.00 & soil_slide \\
\hline rock_slump & missing & 1.00 & rock_slump \\
\hline soil_slump & missing & 1.00 & soil_slump \\
\hline soil_flow & missing & 1.00 & soil_flow \\
\hline
\end{tabular}

\subsection{3 pSoilDepth}

The variable pSoilDepth is based on knowledge from Keefer (1984) and Bommer and Rodriguez (2000). The two fuzzy values lie on either side of 3 meters (Figure 10). This fuzzy threshold represents knowledge regarding whether there is enough soil for a deep-seated failure to occur. If data regarding slope depth is missing CAMEL will assume that Category I landslides in soil are possible. Table 6 shows the associated rules. Notice that the truth-values for all of the fuzzy values are simply passed on to the next rule block, except for "soil_slump.".

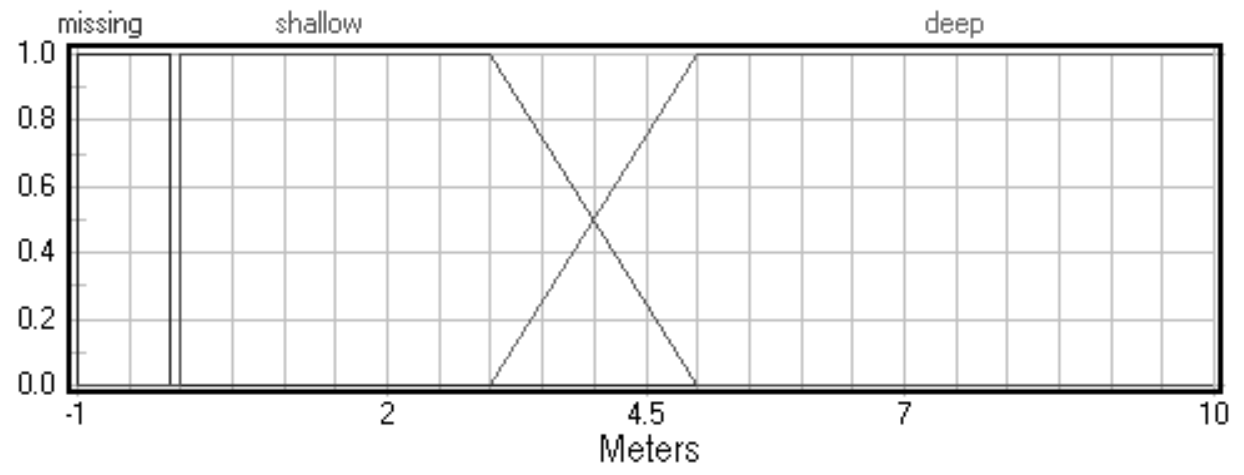

Figure 10. Membership function for pSoilDepth. 
Table 6. Possibility module rule block expressing knowledge about pSoilDepth.

\begin{tabular}{|l|l|l|l|}
\hline \multicolumn{2}{|c|}{ IF } & \multicolumn{2}{c|}{ THEN } \\
\hline LandslideType6 & pSoilDepth & DoS & LandslideType5 \\
\hline \hline soil_slump & deep & 1.00 & soil_slump \\
\hline soil_slump & missing & 1.00 & soil_slump \\
\hline rock_fall & & 1.00 & rock_fall \\
\hline rock_avalanche & & 1.00 & rock_avalanche \\
\hline soil_slide & & 1.00 & soil_slide \\
\hline rock_slump & & 1.00 & rock_slump \\
\hline soil_flow & & 1.00 & soil_flow \\
\hline
\end{tabular}

\subsection{4 pSlopeHeight}

Keefer (1984) observed that for the occurrence of rock avalanches the local slope height must be greater than or equal to 150 meters. This knowledge is reflected in the variable pSlopeHeight (Figure 11), which is comprised of two fuzzy values, "low" and "high." The latter value is defined so that the truth-value is one (1) at a slope height value of 150 meters. The other membership function is simply inverse. The steepness of the membership functions is warranted because 150 meters is an extreme minimum for the avalanche dataset of Keefer (1993). A fuzzy value of "missing" is also possible and is used to assume that rock avalanches are possible in the event of missing slope height data values. Table 7 lists the associated rules and shows that calculations are only performed on the fuzzy value "rock_avalanche." Otherwise, truth values associated with other landslide types are passed to the next rule block.

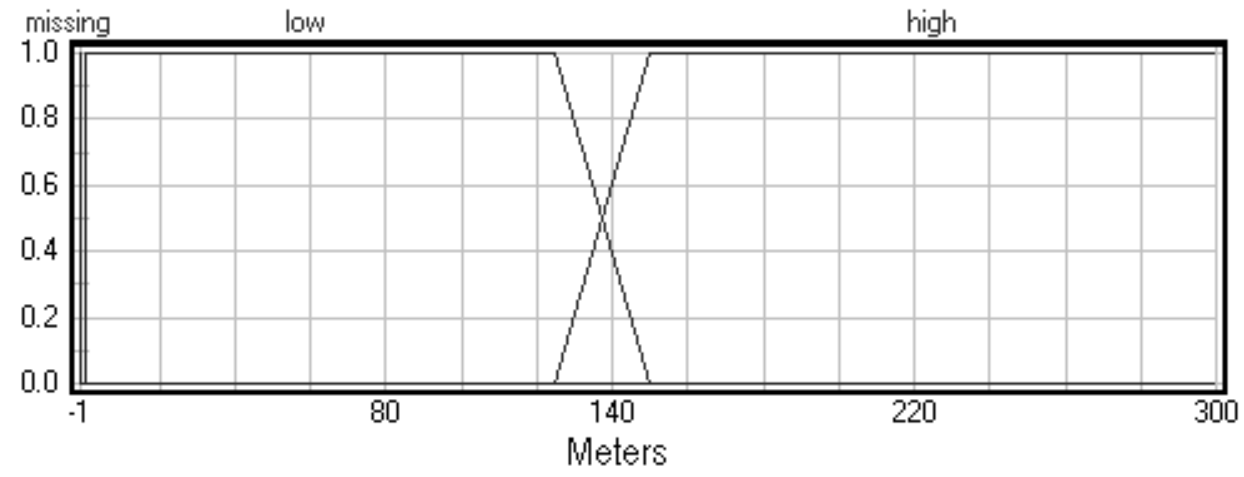

Figure 11. Membership functions for pSlopeHeight. 
Table 7. Possibility module rule block expressing knowledge about pSlopeHeight.

\begin{tabular}{|l|l|l|l|}
\hline \multicolumn{2}{|c|}{ IF } & \multicolumn{2}{|c|}{ THEN } \\
\hline LandslideType5 & pSlopeHeight & DoS & LandslideType4 \\
\hline \hline rock_fall & & 1.00 & rock_fall \\
\hline rock_avalanche & high & 1.00 & rock_avalanche \\
\hline soil_slide & & 1.00 & soil_slide \\
\hline rock_slump & & 1.00 & rock_slump \\
\hline soil_slump & & 1.00 & soil_slump \\
\hline soil_flow & & 1.00 & soil_flow \\
\hline rock_avalanche & missing & 1.00 & rock_avalanche \\
\hline
\end{tabular}

\subsection{5 pMaterialType}

pMaterialType is a nominal variable. It can take on three nominal values; "rock", "soil", and "missing." The variable serves as a binary switch to indicate whether material properties inputs provided (i.e., hGroundClass, defined below) refer to rock or soil. If no indication is provided, the fuzzy system, as shown in the rule block of Table 8 , will apply the same material properties to both rock and soil landslides. Further, both rock and soil landslides are assumed possible.

Table 8. Possibility module rule block expressing knowledge about pMaterialType.

\begin{tabular}{|l|l|l|l|}
\hline \multicolumn{2}{|c|}{ IF } & \multicolumn{2}{l|}{ THEN } \\
\hline LandslideType4 & pMaterialType & DoS & LandslideType3 \\
\hline \hline rock_fall & rock & 1.00 & rock_fall \\
\hline rock_avalanche & rock & 1.00 & rock_avalanche \\
\hline soil_slide & soil & 1.00 & soil_slide \\
\hline rock_slump & rock & 1.00 & rock_slump \\
\hline soil_slump & soil & 1.00 & soil_slump \\
\hline soil_flow & soil & 1.00 & soil_flow \\
\hline rock_fall & missing & 1.00 & rock_fall \\
\hline rock_avalanche & missing & 1.00 & rock_avalanche \\
\hline soil_slide & missing & 1.00 & soil_slide \\
\hline rock_slump & missing & 1.00 & rock_slump \\
\hline soil_slump & missing & 1.00 & soil_slump \\
\hline soil_flow & missing & 1.00 & soil_flow \\
\hline
\end{tabular}

\subsection{6 pMoisture}

pMoisture represents knowledge about different categories of landslides with respect to 
ground moisture content or water table depth. The variable describes the degree to which the analysis layer is saturated, relative to the depth of the layer. pMoisture is conceptually the ratio of the ground water height over the depth of the ground layer under analysis, multiplied by $100 \%$. This is the same definition as the m-value used in infinite slope models typically applied for regional modeling (e.g., Jibson et al., 1998). The knowledge for this variable is based on Keefer (1984). The definition of this variable is shown in Figure 12. The lowest value, "more_than_moist", is related to Category II landslides, assuming that the occurrence of coherent slides require some ground water. The membership function shape indicates that above 0 percent layer saturation some possibility exists. The high value, "about_saturated", is related to rapid soil flows, which require high moisture content (Keefer, 1984). A threshold value of $75 \%$ (indicating truth value of 1) was assumed as a conservative value describing that the entire layer saturated. A higher threshold would reduce possibilities assigned to less-saturated slopes. Looking at the corresponding rules listed in Table 9, notice that "rock_fall", "rock_avalanche", and "soil_slide" are possible regardless of moisture conditions (Keefer, 1993; Keefer, 1984).

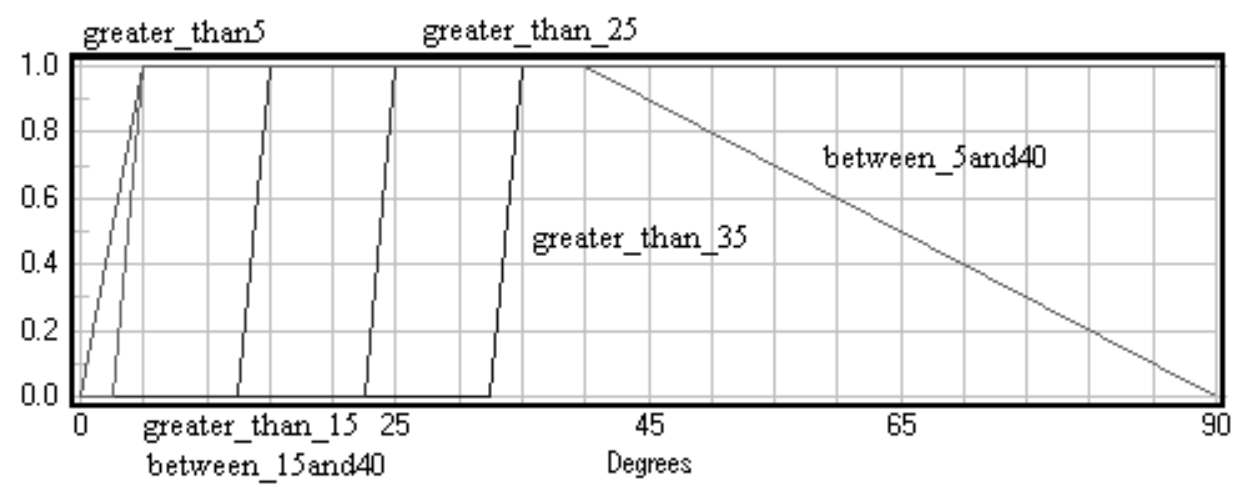

Figure 12. Membership functions of pMoisture. 
Table 9. Possibility module rule block expressing knowledge about pMoisture.

\begin{tabular}{|l|l|l|l|}
\hline \multicolumn{2}{|c|}{ IF } & \multicolumn{2}{c|}{ THEN } \\
\hline LandslideType3 & pMoisture & DoS & LandslideType2 \\
\hline \hline rock_fall & & 1.00 & rock_fall \\
\hline rock_avalanche & & 1.00 & rock_avalanche \\
\hline soil_slide & & 1.00 & soil_slide \\
\hline rock_slump & more_than_moist & 1.00 & rock_slump \\
\hline soil_slump & more_than_moist & 1.00 & soil_slump \\
\hline soil_flow & about_saturated & 1.00 & soil_flow \\
\hline
\end{tabular}

\subsection{7 pSlopeAngle}

Knowledge describing the minimum slope angle required for the occurrence of each landslide type is represented by the variable pSlopeAngle. The five membership functions, shown in Figure 13, are based on Hancox et al. (2002), Rodriguez et al. (1999), and Keefer (1984) (Table 10). In defining the membership function for rapid soil flows, we rounded up the (highest) minimum value found in the literature from 2.3 degrees to 5. The majority of flows in the data set of Keefer (1984) are greater than 5 degrees. This simplifies definition of pSlopeAngle membership functions. Other minimum values attenuate from a truth-value of one (1) at the lowest published minimum slope angle to a truth-value of zero (0) in 2.5 degrees. The fuzzy value "greater_than 15" is applied to Category I landslides in soil and is based on the published values for disrupted soil slides. This means that the possibility for soil avalanches is currently over-predicted by CAMEL because the general minimum slope angle for this landslide type is 35 degrees. Disrupted soil slides are very common (Keefer, 1984); so the variable is designed to reflect this priority. The fuzzy value "greater_than35" is defined based on knowledge about disrupted rock falls and slides. Knowledge about soil block slides was used to define the fuzzy value "between_5and40" and is applied to both soil block slides and soil slumps. The fuzzy value "between_15and40" is based on knowledge about all coherent rock slides. The slope angle maximum of 40 degrees for the Category II landslides is based on Hansen and Franks (1991), Cruden and Varnes (1996), and the data set of Keefer (1984). Because of uncertainty in this maximum, the membership function attenuates slowly until it reaches a truth-value of zero $(0.0)$ at 90 degrees. Table 11 shows the rules dealing with each fuzzy value of pSlopeAngle. 


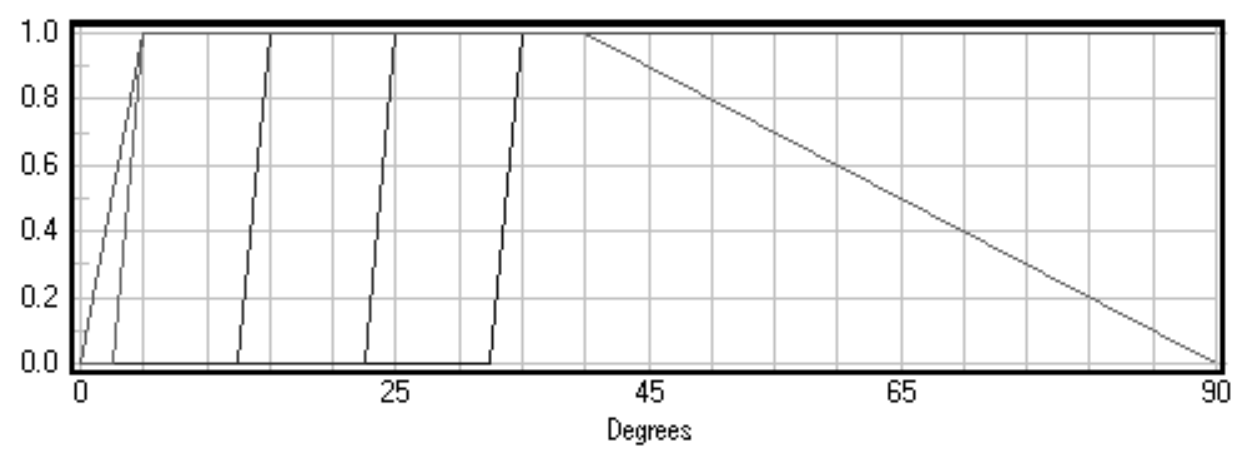

Figure 13. Membership functions for pSlopeAngle.

Table 10. Knowledge about minimum slope angles required for particular types of landslides.

\begin{tabular}{|l|c|c|c|}
\hline \multicolumn{1}{|c|}{ Landslide Type } & \multicolumn{3}{c|}{ Minimum Slope Angle (degrees) } \\
\hline \hline & Keefer (1984) & $\begin{array}{c}\text { Rodriguez et } \\
\text { al. (1999) }\end{array}$ & $\begin{array}{c}\text { Hancox et al. } \\
(2002)\end{array}$ \\
\hline Disrupted rock falls and rock slides & $35-40$ & 35 & 40 \\
\hline Disrupted soil slides/soil falls & $15 / 40$ & 55 & $25-35$ \\
\hline Rock avalanches & 25 & $\mathrm{n} / \mathrm{a}$ & $25-35$ \\
\hline Rock slumps and rock block slides & 15 & 15 & 15 \\
\hline Soil slumps and soil block slides & $5-10$ & 8 & 15 \\
\hline Rapid soil flows & 2.3 & 0 & 2 \\
\hline
\end{tabular}

Table 11. Possibility module rule block expressing knowledge about pSlopeAngle.

\begin{tabular}{|c|l|c|l|}
\hline \multicolumn{2}{|c|}{ IF } & \multicolumn{2}{c|}{ THEN } \\
\hline LandslideType2 & pSlopeAngle & DoS & LandslideType \\
\hline \hline rock_fall & greater_than_35 & 1.00 & rock_fall \\
\hline rock_avalanche & greater_than_25 & 1.00 & rock_avalanche \\
\hline soil_slide & greater_than_15 & 1.00 & soil_slide \\
\hline rock_slump & between_15and40 & 1.00 & rock_slump \\
\hline soil_slump & between_5and40 & 1.00 & soil_slump \\
\hline soil_flow & greater_than5 & 1.00 & soil_flow \\
\hline
\end{tabular}

\subsection{Hazard Module}

The hazard module is illustrated in Figure 14. The module treats each landslide type 
separately in two sub-modules, static susceptibility and seismic hazard to compute different ranges of areal concentration values for each of the six landslide types in CAMEL. After a general introduction of the module below, the three main sets of elements - input variables, output variables, and rule blocks - are specified in detail in the remainder of the section.

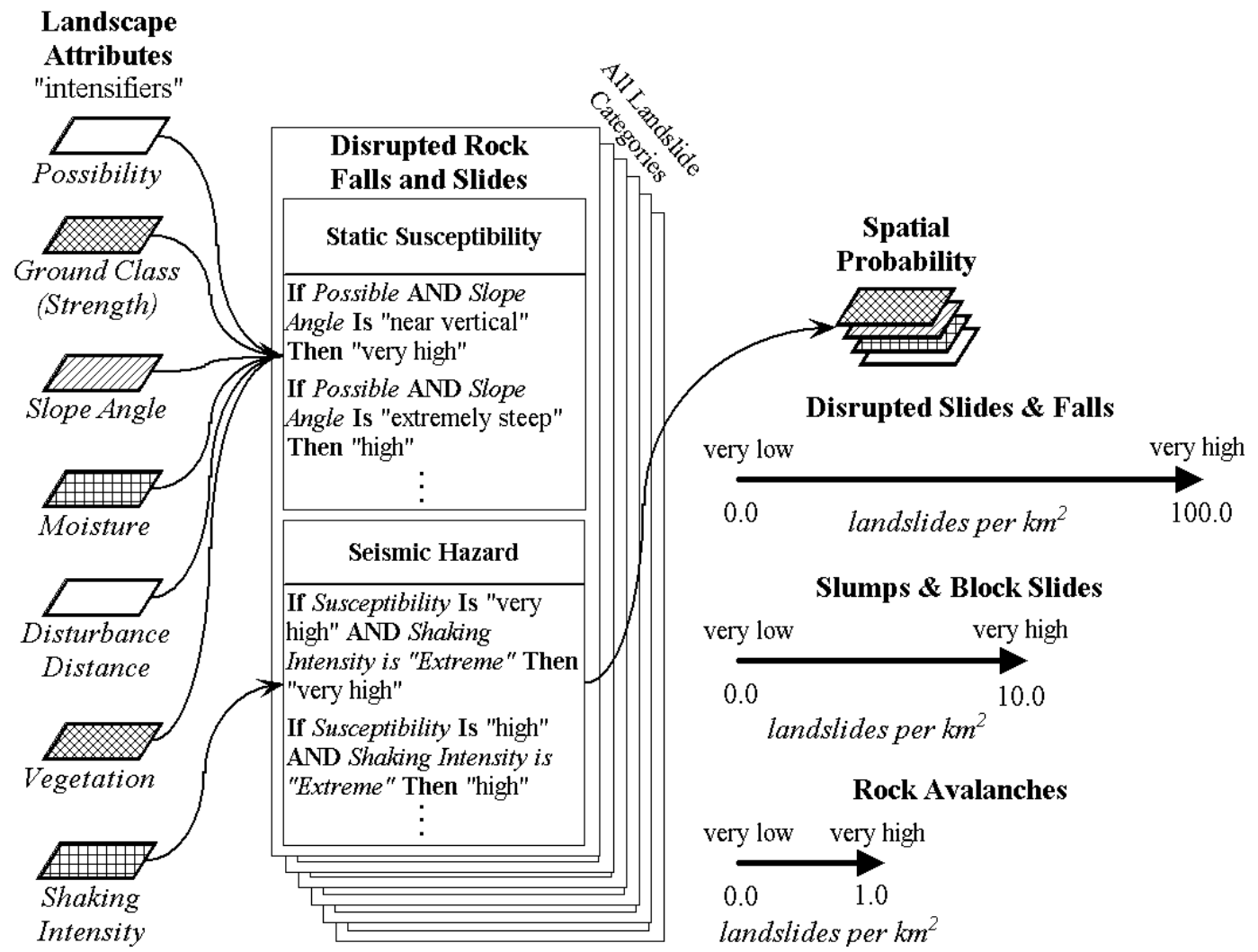

Figure 14. Hazard module of CAMEL.

The susceptibility sub-module is comprised of knowledge about variables independent of earthquake shaking (i.e., static conditions). This knowledge about static susceptibility is represented with two rule blocks. In the first rule block, output variable values monotonically increase with increasing input variable values. In other words, the rules express that for every input increase of one fuzzy value (e.g., "low" to "medium"), the output increases by one fuzzy value. The second rule block of the susceptibility sub-module considers less important variables (or variables associated with poorer detail or quality knowledge), referred to as modifiers in CAMEL. Modifiers contribute to static susceptibility by increasing or decreasing the susceptibility by no more than one fuzzy value, or not at all. For example, if a slope is near an 
undercutting stream and would otherwise have a "medium" static susceptibility, the computed static susceptibility might be "high."

The seismic hazard sub-module considers the earthquake ground-shaking with respect to the static susceptibility computed by the previous sub-module. The seismic hazard sub-module considers all possible combinations of static susceptibility and shaking intensity fuzzy values. The output of the seismic hazard sub-module (and the overall output of CAMEL) is an estimation of areal concentration (landslides per square kilometer) for each landslide type. Importantly, as illustrated in Figure 14, the possible range of concentration values is different for each landslide type. This is described in further detail below.

\subsubsection{Hazard Module Input Variables}

The hazard module is generally, but not directly, based on quantitative knowledge from the scientific literature. Each domain and some specific membership functions are specifically based on elicited knowledge, while other elements are based on design criteria or judgment. Except for specific reasons, membership functions are defined using triangular shapes. In lieu of quantitative data, membership functions were defined using three constraints. First, the domain must be completely specified (or "covered") with membership functions. Second, all neighboring membership functions must intersect at a truth-value of 0.5 . Third, neighboring membership functions should mirror each other. For example, if one membership function exhibits a truthvalue of 0.7 at a particular domain location, the neighboring membership function should give a value of 0.3 at the same location. These criteria help ensure the highest truth-value possible when inputs are uncertain or vague. The criteria further provide that there are no input data in which CAMEL will not compute outputs and prevents dramatically different results over small changes in values.

Table 12 lists all of the input variables for the hazard module. Each variable is defined with the quantitative units, the domain (minimum and maximum values), and the label of the fuzzy values for each variable. Each input variable is specified in further detail below, including utilized sources of knowledge. 
Table 12. CAMEL input variables - variables and modifiers.

\begin{tabular}{|c|c|c|c|c|}
\hline Variable Name & Unit & Min & $\operatorname{Max}$ & $\begin{array}{c}\text { Fuzzy Value } \\
\text { Labels }\end{array}$ \\
\hline hDisturbanceDist & Meters & 0 & 200 & $\begin{array}{l}\text { close } \\
\text { far }\end{array}$ \\
\hline hGroundClass & $\begin{array}{c}\text { Relative } \\
\text { (ratio) units }\end{array}$ & 1 & 5 & $\begin{array}{l}\text { very_good } \\
\text { good } \\
\text { moderate } \\
\text { poor } \\
\text { very_poor }\end{array}$ \\
\hline hMoisture & Percent & 0 & 100 & $\begin{array}{l}\text { very_low } \\
\text { low } \\
\text { medium } \\
\text { high } \\
\text { very_high } \\
\text { not_very_low }\end{array}$ \\
\hline hShakeIntensity & $\begin{array}{l}\text { Shakemap } \\
\text { MMI }\end{array}$ & 4 & 10 & $\begin{array}{l}\text { light } \\
\text { moderate } \\
\text { strong } \\
\text { very_strong } \\
\text { severe } \\
\text { violent } \\
\text { extreme }\end{array}$ \\
\hline hSlopeAngle & $\begin{array}{l}\text { Degrees } \\
\text { from the } \\
\text { horizontal }\end{array}$ & 5 & 65 & $\begin{array}{l}\text { very_gradual } \\
\text { gradual } \\
\text { medium_gradual } \\
\text { medium } \\
\text { medium_steep } \\
\text { steep } \\
\text { very_steep } \\
\text { extremely_steep } \\
\text { nearl_vertical }\end{array}$ \\
\hline hVegetation & Percent & 0 & 100 & $\begin{array}{l}\text { sparse } \\
\text { dense }\end{array}$ \\
\hline
\end{tabular}

\subsubsection{1 hDisturbanceDist}

Conceptually, hDisturbanceDist facilitates representation of morphological factors contributing to slope failure, such as toe erosion by an undercutting stream or an over-steepened road cut (Cruden and Varnes, 1998). Specifically, it is the distance in meters between the slope (however defined) and the identified linear disturbance feature. The domain of the variable and number of fuzzy values is assumed based on inspection of the Loma Prieta landslide inventory of Keefer (1998). Membership function definitions, shown in Figure 15, for the two fuzzy values are based on an assumption that the influence of some linear disturbance is significantly less 
beyond a distance of 100 meters. The s-shape of the membership functions was chosen so that transition between close and far is faster than equivalent straight-line curves. In other words, the fuzziness between the two values is less in comparison to triangular membership functions towards the ends of the domain.

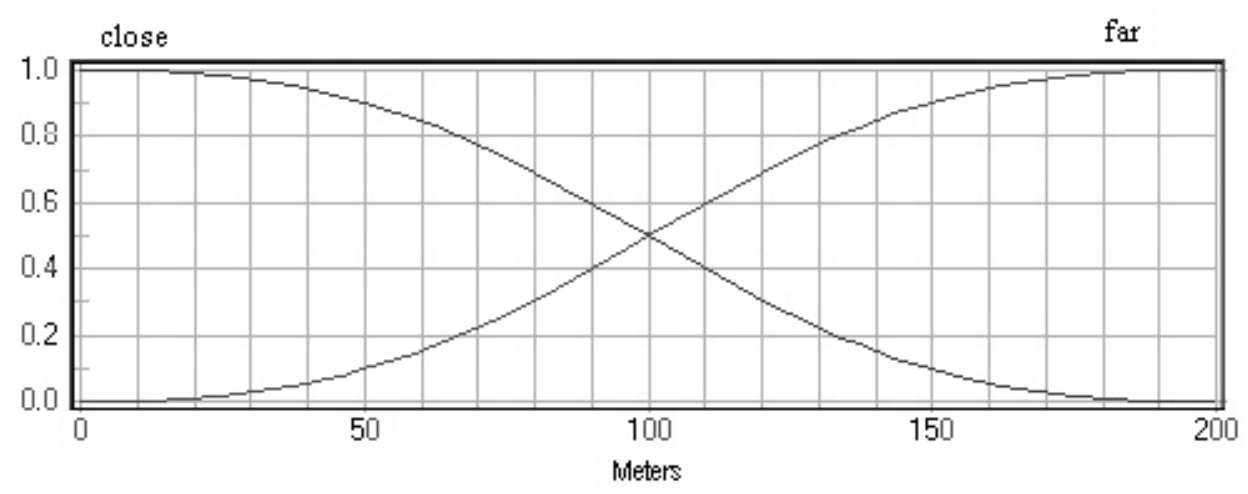

Figure 15. Membership functions of DisturbanceDist.

\subsubsection{2 hGroundClass}

The variable hGroundClass is based on a combination of terminology and knowledge from Hancox et al. (2002) and Keefer (2000) about the relationships between qualitative descriptions of slope material or lithology with earthquake-induced landslide hazard. Figure 16 presents the knowledge from Keefer (2000), describing a monotonic correlation between five linguistic descriptions of lithology that represents a decreasing quality of cementation, induration, or weathering with increasing concentrations of landslides. The ground classes of Hancox et al. (2002) are listed in Table 13. hGroundClass is designed as an index of ground conditions and material properties, such as shear strength and rock jointing, with lower values indicating poorer ground conditions (Figure 17). For this reason, membership function definition was a simple matter of creating identical function shapes for each ground class value. Specifying input data for hGroundClass requires that the user translate data and/or knowledge regarding the ground conditions into one of five categories. This variable represents either rock or soil conditions depending on the value for pMaterialType. 


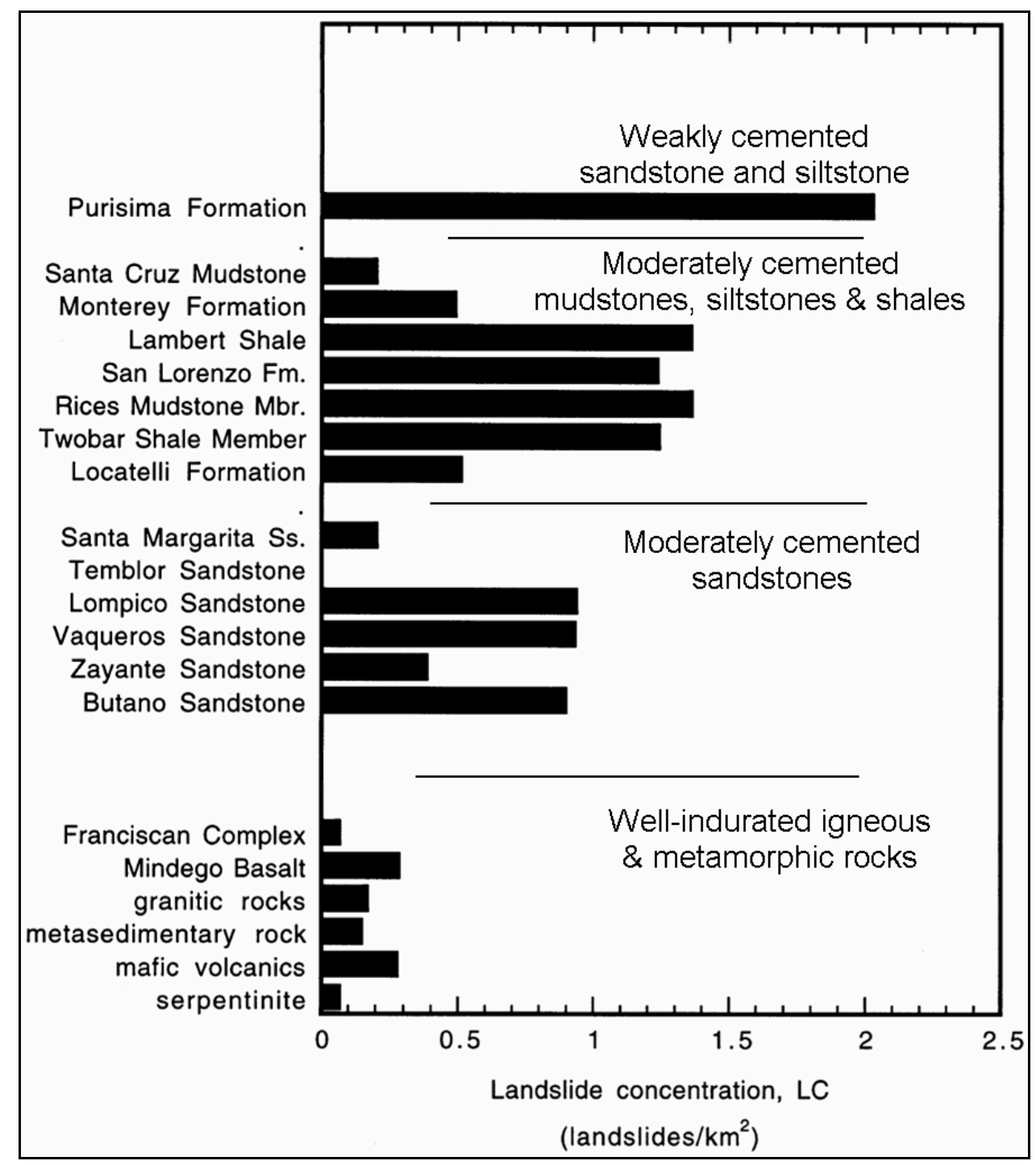

Figure 16. Knowledge describing increasing likelihood of landslides with respect to quality of rock cementation or induration from a study of landslides caused by the 1989 Loma Prieta earthquake (Keefer, 2000). 
Table 13. The ground classes of Hancox et al. (2002).

\section{Class I}

(a) Bedrock - hard to firm rocks, relatively massive (unbedded), both widely and closely jointed, indurated greywacke and granitic rocks, moderately weathered to fresh, with thin $(<1-2 \mathrm{~m})$ surficial colluvial materials, on gentle to moderate slopes $\left(15-30^{\circ}\right)$. Also, firm older alluvial deposits (gravels) forming high terraces (not terrace edges). This class is the benchmark against which other ground classes can be compared.

(b) Supported cut slopes in bedrock; engineered fills on firm ground.

Landslide susceptibility

Low - very low

Class II

Bedrock - well bedded, slightly to moderately weathered Tertiary sandstone, mudstone, and limestone dipping down slope on gentle to moderate slopes $\left(15-30^{\circ}\right.$ dip slopes), with thin regolith and surficial deposits. Also firm to stiff soils.

Landslide susceptibility: Moderate-high

Average change in MM intensity from Class 1 :

$+0.5-1$

Class III

Bedrock - well jointed indurated greywacke and granitic rocks, moderately to highly weathered, with thick $(>5 \mathrm{~m})$ regolith and colluvium on high, steep to very steep (say $35-50^{\circ}$ ) slopes, and on high narrow ridges (near and far field). Also low gravel banks and terrace edges, scree deposits, and slopes and cuts formed in loose unconsolidated deposits.

Landslide susceptibility:

High-Very High

Average change in MM intensity from Class I:

$+1-1.5$

Class IV

(a) Areas of very steep $\left(>45^{\circ}\right)$ natural slopes (such as cliffs escarpments, gullies, and gorges) in hard, jointed rocks, weak Tertiary rocks, and also weakly-cemented Quaternary deposits (loess, pumice).

(b) Unsupported high $\left(>3-6 \mathrm{~m}\right.$ ), very steep (say $>60^{\circ}$ ) cuts and excavations in harder bedrock and soft rocks, especially those cuts capped with $1.3 \mathrm{~m}$ of soils and regolith deposits, and not designed to withstand the effects of seismic shaking.

Landslide susceptibility:

Average change in MM intensity from Class l:

High-very high

$+1 \cdot 2$

Class V

Loose, saturated, unconsolidated, fine-grained, alluvial, estuarine and marine deposits (fine sand, silt), and other soft sediments, nonengineered fills and reclamations on flat, low -lying terrain and gentle slopes $\left(<10^{\circ}\right)$

Landslide susceptibility:

High- very high

Average change in MM intensity from Class I - Near field ": +0.5-1

Low frequency shaking - Far field ${ }^{1}>M 7.2$ earthquakes): $\quad+1-3$ 


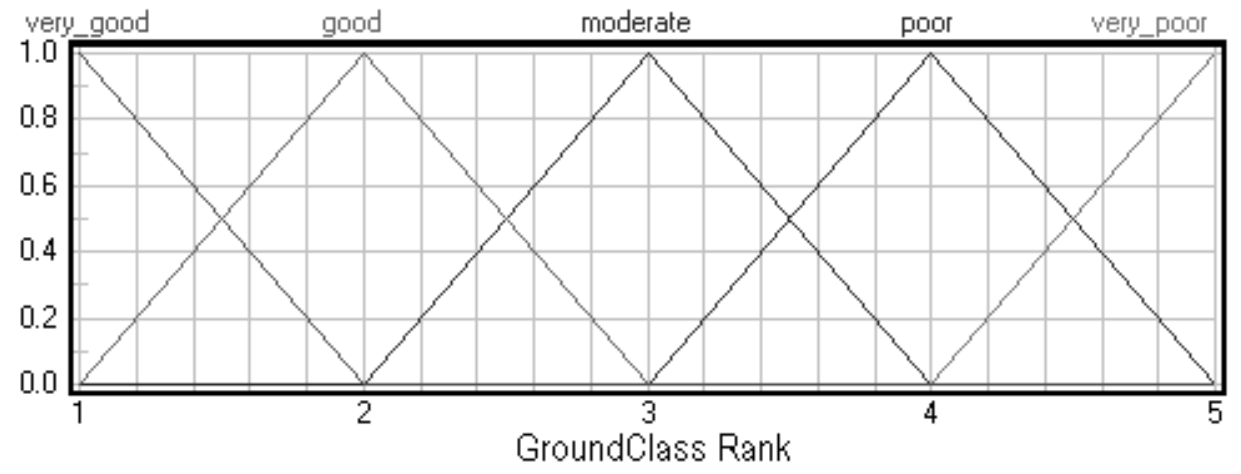

Figure 17. Membership functions of hGroundClass.

\subsubsection{3 hMoisture}

Conceptually, hMoisture is defined in the same manner as pMoisture. The variable describes the degree to which the analysis ground layer is saturated, relative to the depth of the layer. hMoisture is the ratio of the ground water height over the depth of the ground layer under analysis, multiplied by $100 \%$. The domain is from 0 to $100 \%$ layer saturation. Just as hGroundClass, the five fuzzy values for hMoisture were determined by dividing the domain into five categories defined by symmetrically shaped membership functions (Figure 18).

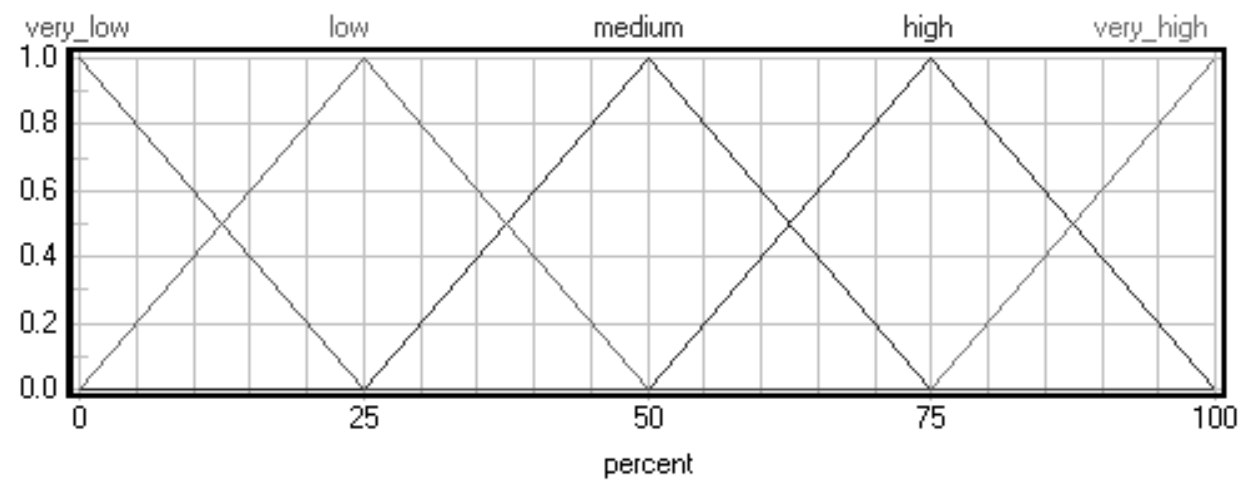

Figure 18. Membership functions of hMoisture.

\subsubsection{4 hShakelntensity}

The variable $h$ ShakeIntensity represents knowledge about earthquake shaking intensity. The variable is simply a fuzzy translation of the MMI scale (considering the values between and including 4 and 10), with a symmetrical membership function located at each MMI value (Figure 
19). The fuzzy value labels are based on the ShakeMap terms of Wald et al. (1999). No fuzzy values greater than 10 are defined because ShakeMap does not provide these values and little data exists on landslides related to such large intensities.

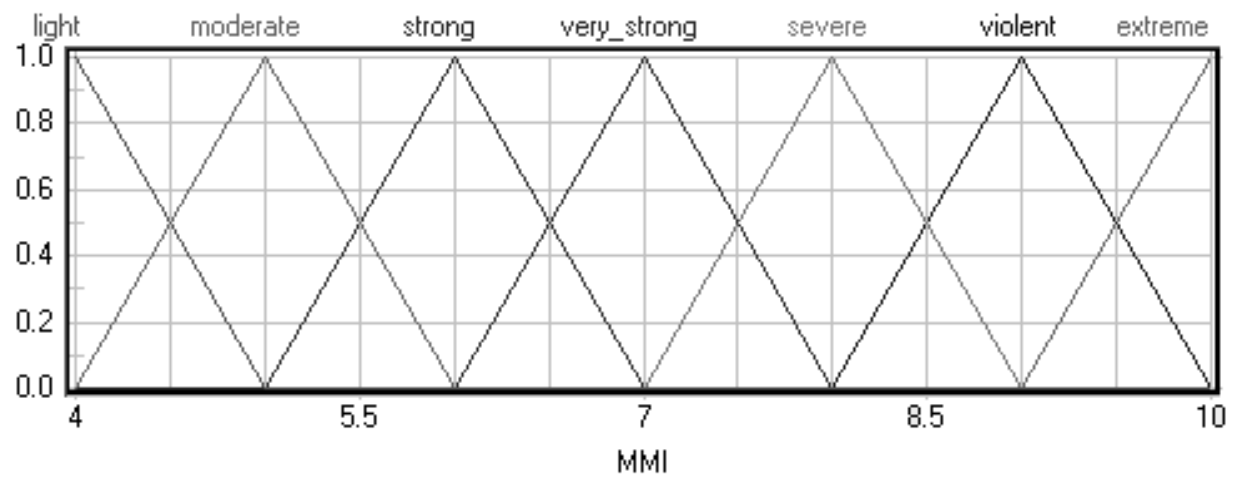

Figure 19. Membership functions of hShakeIntensity.

\subsubsection{5 hSlopeAngle}

Knowledge about the influence of increasing slope angle is represented by hSlopeAngle (Figure 20). The units are degrees from the horizontal — the same as pSlopeAngle. The minimum value ( 5 degrees) defining the domain is based on the indicator threshold for rapid soil flows and soil slumps. For slope values less than 5 degrees, no landslide type is possible with respect to the current specifications of CAMEL. A maximum value of 65 degrees is used because this value typically served as the maximum value analyzed in studies refereed to for knowledge elicitation. For example, Hancox et al. (2002) relates a cutoff 65 degrees with "High to Very High" susceptibility. To be logically consistent, each membership function defining pSlopeAngle has a corresponding membership function in hSlopeAngle. The membership function located at 20 degrees is to provide five functions between 15 and 40, corresponding to the possible slope values for rock slumps and block slides. Membership functions located at angles greater than 40 are spaced at equal intervals up to 65 degrees. The asymmetric triangle-shaped membership functions provide smooth transitions between fuzzy values. If symmetrical trapezoid shapes were used to define hSlopeAngle, output values for CAMEL would be the same for some ranges of slope angles because a trapezoidal shape defines a truth value of one (1) for an interval rather than a point. 


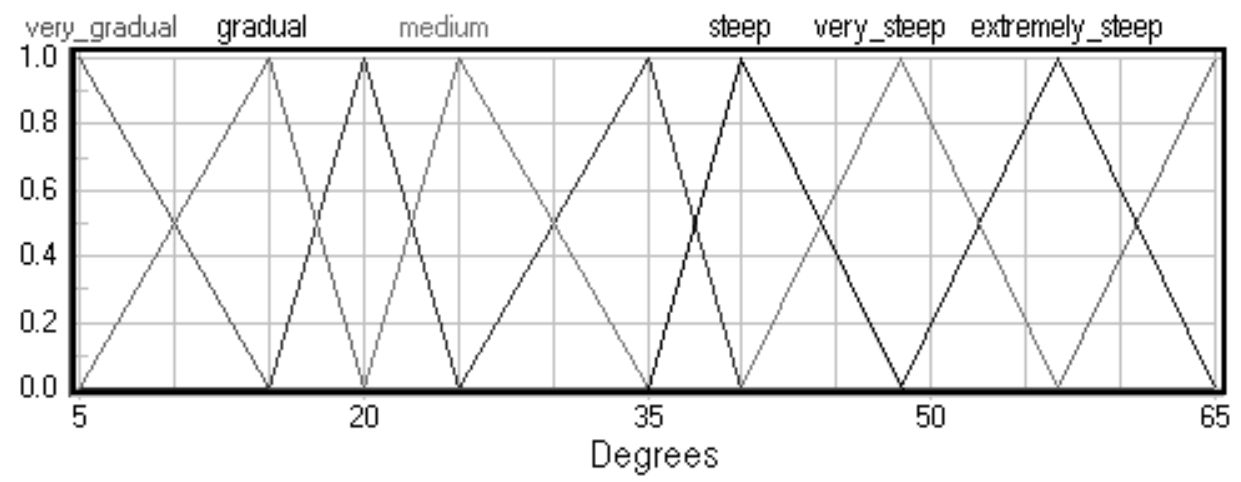

Figure 20. Membership functions of hSlopeAngle.

\subsubsection{6 hVegetation}

Little specific knowledge about the influence of vegetation on the different types of earthquake-induced landslides exists in the literature beyond the decision tree for assessing disrupted rock fall and slide susceptibility of Keefer (1993). For this reason, a simple definition for $h$ Vegetation was assumed (Figure 21). Conceptually, the variable refers to the vegetation coverage (density) of a given area, expressed as a percent of the area. Two fuzzy values, "sparse" and "dense" are defined, with 50 percent serving as the decision boundary. The utility of this variable may be limited in the current version of the model, but does not degrade the performance of the model because it is an optional variable.

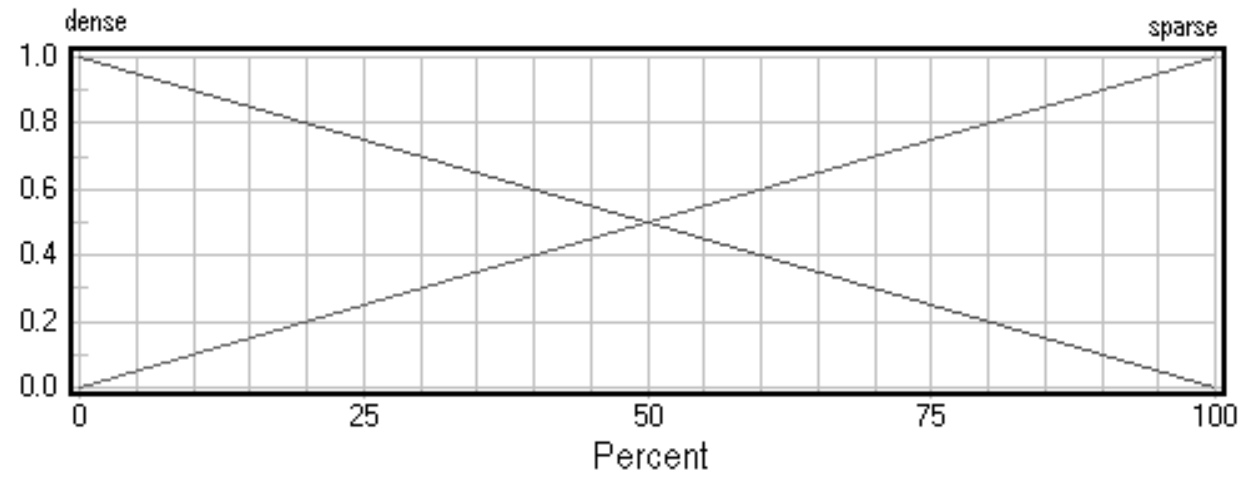

Figure 21. Membership functions of $h$ Vegetation. 


\subsubsection{Output Variables - Landslide Concentration}

The variables listed in Table 14 are the outputs associated with the hazard module of CAMEL. Each of the six landslide types is associated with four output variables. These correspond to two seismic hazard output variables — one that is defuzzified and a nominal variables that isn't (e.g., RAvSeismic and RAvSeismicFz) —and, similarly, two static susceptibility output variables (e.g., RAvStatic and RAvStaticFz). (FuzzyTech ${ }^{\mathrm{TM}}$, the software used to implement CAMEL, requires specification of the defuzzification method as part of defining variables and so two separate variables must be defined if one wants to view outputs both as a defuzzified value and as fuzzy sets.) The fuzzy values for all of the output variables are the same, but are associated with a different domain and membership function locations (latter three columns of Table 14). Definition of the domains and membership locations are described in more detail below. 
Table 14. Specifications of output variables for the hazard module.

\begin{tabular}{|c|c|c|c|}
\hline Variable Name & $\begin{array}{c}\text { Fuzzy Value } \\
\text { Labels }\end{array}$ & \begin{tabular}{|c|} 
Location of \\
Membership \\
Function \\
Centers \\
$\left(\ln 1 \mathrm{~s} / \mathrm{km}^{2}\right)$ \\
\end{tabular} & $\begin{array}{c}\text { Location of } \\
\text { Membership } \\
\text { Function } \\
\text { Centers } \\
\left(1 \mathrm{~s} / \mathrm{km}^{2}\right) \\
\end{array}$ \\
\hline $\begin{array}{l}\text { RAvSeismic } \\
\text { RAvStatic } \\
\text { RAvSeismicFz } \\
\text { RAvStaticFz }\end{array}$ & $\begin{array}{l}\text { very_low } \\
\text { low } \\
\text { medium } \\
\text { high } \\
\text { very_high }\end{array}$ & $\begin{array}{c}-4.605 \\
3.454 \\
-2.303 \\
-1.151 \\
0\end{array}$ & $\begin{array}{c}0.01 \\
0.0315 \\
0.1 \\
0.316 \\
1\end{array}$ \\
\hline $\begin{array}{l}\text { RFallSeismic } \\
\text { RFallStatic } \\
\text { RFallSeismicFz } \\
\text { RFallStaticFz }\end{array}$ & $\begin{array}{l}\text { very_low } \\
\text { low } \\
\text { medium } \\
\text { high } \\
\text { very_high }\end{array}$ & $\begin{array}{l}-2.303 \\
-0.576 \\
1.151 \\
2.878 \\
4.605\end{array}$ & $\begin{array}{c}0.1 \\
0.562 \\
3.161 \\
17.779 \\
100\end{array}$ \\
\hline $\begin{array}{l}\text { SFallSeismic } \\
\text { SFallStatic } \\
\text { SFallSeismicFz } \\
\text { SFallStaticFz }\end{array}$ & $\begin{array}{l}\text { very_low } \\
\text { low } \\
\text { medium } \\
\text { high } \\
\text { very_high }\end{array}$ & $\begin{array}{c}-2.303 \\
-0.576 \\
1.151 \\
2.878 \\
4.605\end{array}$ & $\begin{array}{c}0.1 \\
0.562 \\
3.161 \\
17.779 \\
100\end{array}$ \\
\hline $\begin{array}{l}\text { RSlumpSeismic } \\
\text { RSlumpStatic } \\
\text { RSlumpSeismicFz } \\
\text { RSlumpStaticFz }\end{array}$ & $\begin{array}{l}\text { very_low } \\
\text { low } \\
\text { medium } \\
\text { high } \\
\text { very_high }\end{array}$ & $\begin{array}{c}-2.303 \\
-1.151 \\
0 \\
1.151 \\
2.303\end{array}$ & $\begin{array}{c}0.1 \\
0.316 \\
1 \\
3.161 \\
10\end{array}$ \\
\hline $\begin{array}{l}\text { SSlumpSeismic } \\
\text { SSlumpStatic } \\
\text { SSlumpSeismicFz } \\
\text { SSlumpStaticFz }\end{array}$ & $\begin{array}{l}\text { very_low } \\
\text { low } \\
\text { medium } \\
\text { high } \\
\text { very_high }\end{array}$ & $\begin{array}{c}-2.303 \\
-1.151 \\
0 \\
1.151 \\
2.303\end{array}$ & $\begin{array}{c}0.1 \\
0.316 \\
1 \\
3.161 \\
10\end{array}$ \\
\hline $\begin{array}{l}\text { SFlowSeismic } \\
\text { SFlowStatic } \\
\text { SFlowSeismicFz } \\
\text { SFlowStaticFz }\end{array}$ & $\begin{array}{l}\text { very_low } \\
\text { low } \\
\text { medium } \\
\text { high } \\
\text { very_high }\end{array}$ & $\begin{array}{c}-2.303 \\
-1.151 \\
0 \\
1.151 \\
2.303 \\
\end{array}$ & $\begin{array}{c}0.1 \\
0.316 \\
1 \\
3.161 \\
10\end{array}$ \\
\hline
\end{tabular}

Because the best knowledge available is for disrupted rock falls and slides, the domain and membership functions for this type of landslide were defined based on the quantitative knowledge available and then scaled down to describe the other landslide types. For disrupted rock fall and slides, the maximum possible rock fall concentration is defined as 100 landslides per square kilometer $\left(1 \mathrm{~s} / \mathrm{km}^{2}\right)$ and the minimum at $0.1 \mathrm{ls} / \mathrm{km}^{2}$. This maximum is an assumed value based on the high concentrations (about $741 \mathrm{~s} / \mathrm{km}^{2}$ ) measured by Parise and Jibson (2000) for the 1994 Northridge, CA earthquake. The highest category in the rock fall decision tree of Keefer 
(1993) is only $16 \mathrm{ls} / \mathrm{km}^{2}$.

A natural log scale was used for the domain of all output variables of the hazard module for two reasons. First, an arithmetic scale would result in the lower membership functions being clustered tightly together, giving too much influence to the higher fuzzy values during the defuzzification process. With a natural log scale, the peak of each membership function can be located at equal intervals. This leads to the second reason for the log scale, being that the 3.16 $\mathrm{ls} / \mathrm{km}^{2}$ geometric increase between each fuzzy value is similar to the four-fold increase between the concentration categories of Keefer (1993). The use of a log scale creates the problem that landslide concentration output values will always be greater than zero (0). However, from an implementation perspective, the variable LandslideType can be used to ensure that, if the possibility is zero (0) (or some other very small value), the reported landslide concentration is also zero (0).

In the latter three columns of Table 14, the fuzzy value labels are given with the respective location within the domain of the membership function peaks, expressed both as a natural $\log$ and arithmetically $\left(\mathrm{ls} / \mathrm{km}^{2}\right)$. CoM (Center of Maximum) defuzzification is used, which only requires specification of the peak (or center) of each membership function. Notice that all of the peak locations are located at equal intervals on the logarithmic domain.

The remaining output variables were specified by scaling the variable definition of RFallSeismic (etc.) based on the historical abundance observations in Keefer (1984) (Table 15). Keefer (1984) noted roughly a magnitude difference between several groupings of landslide types, with rock avalanches being least abundant and rock falls being most abundant. Because disrupted soil slides are very abundant, the corresponding variables (SFallSeismic etc.) were specified identically to the variables for rock falls and disrupted rock slides. Soil slumps and block slides are abundant according to Keefer (1984), with total numbers being about a magnitude less than for rock falls and disrupted rock slides. Therefore, the variables defined for rock falls were scaled down by a factor of 10, making the maximum possible soil slump and block slide concentration $10 \mathrm{ls} / \mathrm{km}^{2}$. According to Table 15 these landslide types are either a magnitude or two less common than soil slumps and block slides, to simplify variable design, the same specification was given to rapid soil flows and rock slumps and block slides. Lastly, rock avalanches are three 
magnitudes less common than rock falls and disrupted rock slides. However, to allow for a higher maximum than indicated in Keefer, 1984, 1 1s $/ \mathrm{km}^{2}$ is specified for the maximum possible rock avalanche concentration.

Table 15. Relative abundance of earthquake-induced landslides from $\mathbf{4 0}$ historical, worldwide earthquakes after Keefer (1984). Landslide types are listed in order of decreasing total numbers.

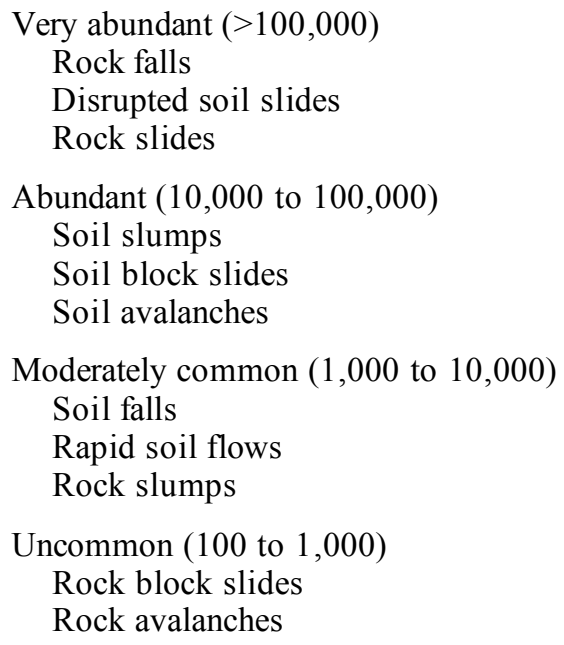

\subsubsection{Hazard Module Rule Blocks}

For each of the six landslide types in CAMEL, there are three associated rule blocks that make up the respective component of the hazard module and sub-modules. Thus, there are a total of 18 rule blocks in the hazard module - six static susceptibility rule blocks, six optional "modifier" rule blocks, and six seismic hazard rule blocks. The variables considered within the static susceptibility and modifier rule-blocks can be different between the respective landslide types. The decisions of what role each variable plays with respect to the different landslide types were made based on the results of knowledge elicitation. Table 16 lists each landslide type considered in CAMEL together with which variables are treated as variables, modifiers or not at all. pShakeIntensity is considered for all landslide types. For brevity, only the rule blocks for disrupted rock slides and falls are presented below. Except for input variables considered, the rule composition and design choices are the same. All of the rule blocks that comprise the hazard module of CAMEL are presented in Appendix A. 
Table 16. Input variables considered for each landslide type within the hazard module of CAMEL.

\begin{tabular}{|l|l|l|}
\hline \multicolumn{1}{|c|}{ Landslide Type } & $\begin{array}{c}\text { Static } \\
\text { Susceptibility }\end{array}$ & \multicolumn{1}{c|}{ Modifiers } \\
\hline \hline Rock Avalanches & $\begin{array}{l}\text { hGroundClass } \\
\text { hSlopeAngle }\end{array}$ & hDisturbanceDist \\
\hline $\begin{array}{l}\text { Disrupted Rock Slides } \\
\text { and Falls }\end{array}$ & $\begin{array}{l}\text { hGroundClass } \\
\text { hSlopeAngle }\end{array}$ & $\begin{array}{l}\text { hDisturbanceDist } \\
\text { hMoisture } \\
\text { hVegetation }\end{array}$ \\
\hline $\begin{array}{l}\text { Disrupted Soil Slides, } \\
\text { Falls, and Avalanches }\end{array}$ & $\begin{array}{l}\text { hGroundClass } \\
\text { hSlopeAngle }\end{array}$ & $\begin{array}{l}\text { hDisturbanceDist } \\
\text { hMoisture } \\
\text { hVegetation }\end{array}$ \\
\hline $\begin{array}{l}\text { Rock Slumps and } \\
\text { Block Slides }\end{array}$ & $\begin{array}{l}\text { hGroundClass } \\
\text { hSlopeAngle } \\
\text { hMoisture }\end{array}$ & $\begin{array}{l}\text { hVegetation } \\
\text { hVanceDist }\end{array}$ \\
\hline $\begin{array}{l}\text { Soil Slumps and Block } \\
\text { Slides }\end{array}$ & $\begin{array}{l}\text { hSlopeAndClass } \\
\text { hMoisture }\end{array}$ & $\begin{array}{l}\text { hDisturbanceDist } \\
\text { hVegetation }\end{array}$ \\
\hline Rapid Soil Flows & $\begin{array}{l}\text { hGroundClass } \\
\text { hSlopeAngle }\end{array}$ & $\begin{array}{l}\text { hDisturbanceDist } \\
\text { hMoisture } \\
\text { hVegetation }\end{array}$ \\
\hline
\end{tabular}

\subsubsection{Static Susceptibility Rule Block}

The static susceptibility rule-block for disrupted rock slides and rock falls is given in Table 17. Within the rule block, hGroundClass and hSlopeAngle, are considered with a separate set of independent rules following the Combs and Andrews (1998) method for single-input, single-output rule composition. That is, hGroundClass and hSlopeAngle are never considered together in a single rule. The Combs and Andrews (1998) method uses a union rule configuration (i.e., an implicit OR between rules) in place of the more common intersection rule configuration where every combination of fuzzy values between input variables is explicitly mapped using AND. 
Table 17. Static susceptibility rule block for disrupted rock slides and falls.

\begin{tabular}{|l|l|l|l|l|}
\hline \multicolumn{3}{|c|}{ IF } & \multicolumn{2}{c|}{ THEN } \\
\hline hGroundClass & LandslideType & hSlopeAngle & DoS & RFallStaticInt \\
\hline \hline very_good & rock_fall & & 0.80 & very_low \\
\hline good & rock_fall & & 0.80 & low \\
\hline moderate & rock_fall & & 0.80 & medium \\
\hline poor & rock_fall & & 0.80 & high \\
\hline very_poor & rock_fall & & 0.80 & very_high \\
\hline & rock_fall & medium_steep & 0.20 & very_low \\
\hline & rock_fall & steep & 0.20 & low \\
\hline & rock_fall & very_steep & 0.20 & medium \\
\hline & rock_fall & extremely_steep & 0.20 & high \\
\hline & rock_fall & nearly_vertical & 0.20 & very_high \\
\hline
\end{tabular}

While the variables are treated separately, the variable LandlideType (from the possibility module) is aggregated using the PROD operator with the particular susceptibility in all of the rules. (PROD is used throughout the hazard module.) This is the element of the design that passes on the determined possibility of each landslide type to the assessment of areal concentration. If the possibility is zero (0), the concentration is also zero (0). If there is some positive possibility, then the landslide concentration is also positive, although it may be very low. An effect of this design is that computed landslide concentrations will be the same whether the possibility is one (1) or less than one (1), but greater than zero (0). However, the lower possibility value is expressed by the fuzzy output variables (e.g., RFallSeismicFz and RFallStaticFz).

Combination of the output fuzzy values (i.e., based on hGroundClass and hSlopeAngle, respectively) is done using the BSUM operator. Using BSUM facilitates different weighting of the variables using a DoS weight. Currently in CAMEL, hGroundClass is weighted higher (DOS $=0.8$ ) than hlopeAngle (DOS =0.2). The weights chosen are arbitrary and can be modified in the future to fine-tune the performance of CAMEL. This choice was made because there is little quantitative knowledge in the scientific literature expressing that rock fall susceptibility increases beyond about 45 degrees. Conversely, this is what limit-equilibrium models describes. So another, perhaps more insightful, interpretation of the use of different DoS weights is that there is more confidence in the knowledge expressed by the hGroundClass rules than for the 
hSlopeAngle rules.

A design limitation encountered for the other landslide types (i.e., not illustrated in Table 17 ) is that for several static susceptibility rule-blocks there are more hSlopeAngle fuzzy values than there are output fuzzy values (always five). This is the result of the different landslide types being possible for a different range of pSlopeAngle values and the design criteria that there be at least five values being available within the valid range for each landslide type. This is dealt with in two different ways within the static susceptibility rule blocks. Either the output values are increased by one fuzzy value for every two increasing fuzzy values of hSlopeAngle or increasing DoS weights are used to differentiate the "very_high" output fuzzy value.

\subsubsection{Modifiers Rule Block}

The rules for the modifiers sub-module are shown in Table 18. (The rules for RFallStaticFz are identical.) The concept behind the modifiers is that if some modifier condition exists, then the static susceptibility determined in the previous rule block should be increased or decreased by one fuzzy value, depending. Further, input of data for modifier variables is optional. There are 20 rules within the rule block. Five rules do not consider any of the three modifiers and simply pass on the fuzzy value determined in the previous rule block. These rules give greater weight to the knowledge from the previous rule-block. The remaining 15 rules consider every combination of the three modifiers (one fuzzy value each) with the five fuzzy values of RFallStaticInt - an intermediate variable, having the same specification as RFallStatic. Each modifier rule is given a DoS weight of $1 / \mathrm{n}$, where $\mathrm{n}$ is the number of modifiers considered for the particular output fuzzy value. Using BSUM aggregation, the effect of the three modifiers is cumulative. Without this design, having more than one modifier with a truth value of one (1) would have the same effect as a single modifier having truth value of one (1). To ensure outputs are monotonic, each fuzzy value of each modifier must be considered, even if the modifier does not affect the output from the previous rule block-for example, the first rule in Table 18. 
Table 18. Modifiers rule block for disrupted rock slides and falls.

\begin{tabular}{|l|l|l|l|l|l|}
\hline \multicolumn{2}{|c|}{ IF } & \multicolumn{2}{c|}{ THEN } \\
\hline hDisturbanceDist & hMoisture & RFall_taticInt & hVegetation & DoS & \multicolumn{1}{c|}{ RFall_tatic } \\
\hline & & very_low & dense & 1.00 & very_low \\
\hline & & low & dense & 1.00 & very_low \\
\hline & & very_low & & 1.00 & very_low \\
\hline & & medium & dense & 0.34 & low \\
\hline & & & & 0.34 & low \\
\hline close & not_very_low & very_low & & 1.00 & low \\
\hline & & low & & 0.34 & low \\
\hline & & very_low & & 0.34 & medium \\
\hline & & high & dense & 0.34 & medium \\
\hline close & not_very_low & low & & 1.00 & medium \\
\hline & & medium & & 0.34 & medium \\
\hline & & low & & 0.34 & high \\
\hline close & & very_high & dense & 0.34 & high \\
\hline & & medium & & 1.00 & high \\
\hline & not_very_low & high & & 0.34 & high \\
\hline & & medium & & 0.34 & very_high \\
\hline close & not_very_low & very_high & & 0.34 & very_high \\
\hline close & not_very_low & high & & 1.00 & very_high \\
\hline & & very_high & & 0.34 & very_high \\
\hline & & high & & 0.34 & very_high \\
\hline
\end{tabular}

A limitation of the design of the modifier rule block using the Combs and Andrews (1998) method is that for a given fuzzy value (assuming data is provided) two rules will be considered: (1) the rule that considers the particular modifier (e.g., hVegetation) and the variable used to pass the outputs from the susceptibility rule block (e.g., RFallStaticInt) and (2) the rule that simply passes on the output from the susceptibility rule block. If the latter rule(s) were not specified, no rules would result in a non-zero truth value in cases when no data is provided for any of the modifiers. As designed, the result however is that a modifier does not have the effect of increasing the susceptibility by one full fuzzy value, but rather some partial amount.

\subsubsection{Seismic Hazard Rule Block}

Figure 22 presents all of the rules that comprise the seismic hazard rule block for disrupted rock slides and falls. The rules are the same for both RFallSeismic and RFallSeismicFz. Also, because the historical abundance is nearly the same for rock and soil (Keefer, 1984), the 
rules are the same for SFallSeismic and SFallSeismicFz. The rule block for determining the final landslide concentration (and associated uncertainty) is different than the previous two rule blocks in that it considers the intersection of all combinations of fuzzy values between RFallStatic and hShakeIntensity. The Combs and Andrews (1998) method is not to be appropriate for this rule block because more control is required to enforce limiting conditions. That is, for example, if RFallStatic is "very_low", the final hazard must be "very_low." With the Combs and Andrews (1998) method, if the hShakeIntensity happened to be "extreme", it would influence the output excessively and result in a final concentration value significantly greater than the membership function location for "very_low" of RFallSeismic.

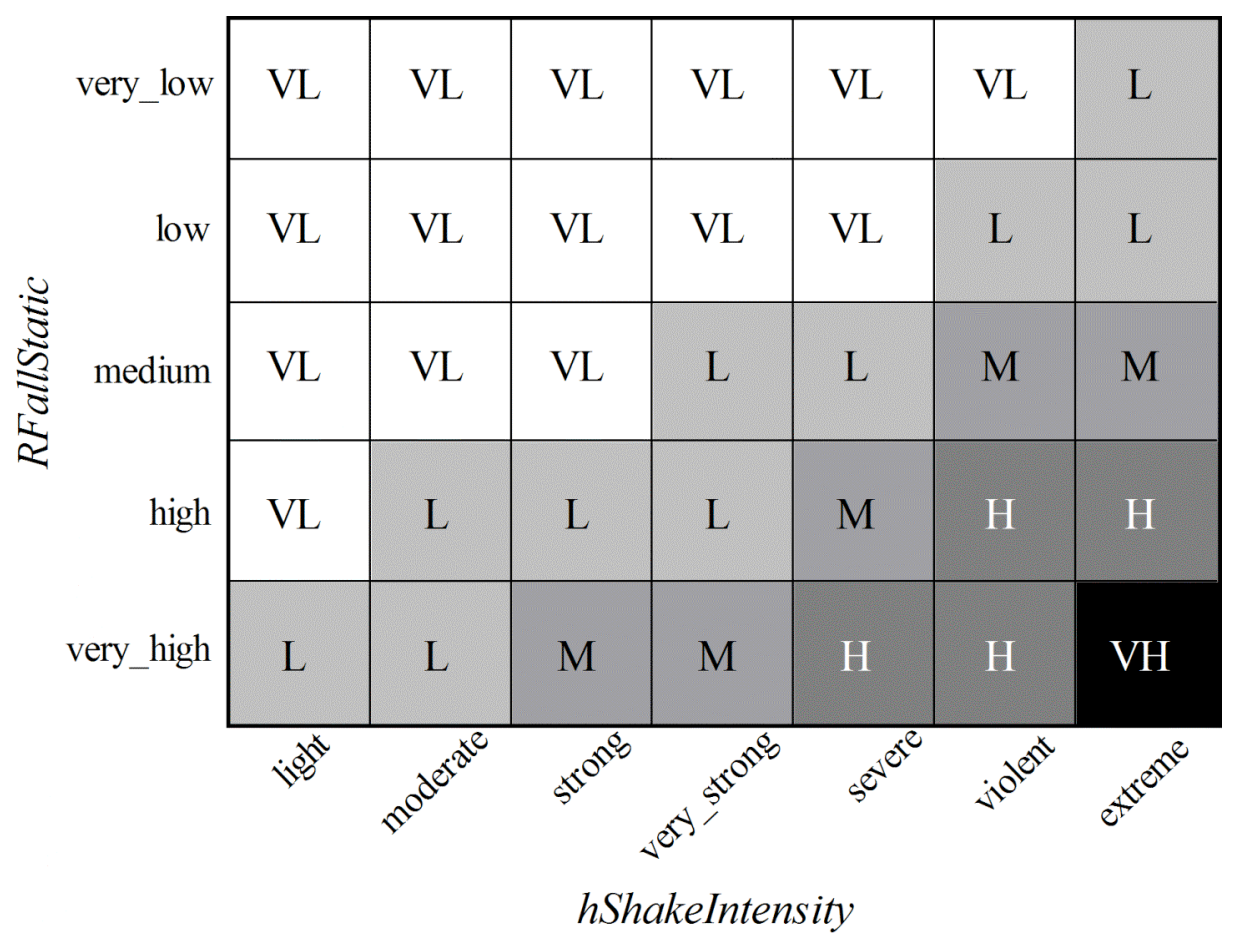

Figure 22. Seismic variables rule block for disrupted rock slides and falls. VL = very_low, $\mathrm{L}=$ low, $\mathrm{M}=$ medium, $\mathrm{H}=$ high, $\mathrm{VH}=$ very_high.

All rules within the rule block are given equal weight $(\mathrm{DoS}=1.0)$. The two inputs are combined using the PROD operator. BSUM is used for output aggregation to favor the output fuzzy values with the most rules. Thus, BSUM favors "very_low" (and so on, favoring "very_high" the least). If MAX output aggregation was used, the one rule mapping to 
"very_high" would have equal weight to all of the rules mapping to "very_low.". All of the quantitative landslide concentrations associated with "very_high" (corresponding to 100 ls $/ \mathrm{km}^{2}$ for disrupted slides and falls) should be a rare occurrence because of the historical infrequency of such concentrations.

The knowledge available within the scientific literature regarding the intersection of static susceptibility and shaking intensity is not detailed enough for a one-to-one translation into fuzzy rules. In light of this, initial rules were specified based on constraints described in the literature, in addition to several pre-defined rule-specification criteria. The most general, but highest priority, specification criterion is that the fuzzy surface represented by the shades of gray in Figure 34 should be smooth and monotonic. That is, the output fuzzy values of adjacent rules should never be more than one fuzzy value apart (e.g., "low" to "medium"). The highest output fuzzy value ("very_high" landslide concentration) should only be assigned to the "very_high" static susceptibility. A "very_high" landslide concentration, in fact, represents an extreme case that is historically very rare. A "very_low" landslide concentration should always be assigned to "very_low" susceptibility. A landslide concentration of "very_low" should be assigned to hShakeIntensity fuzzy values equal to or less than the corresponding indicator threshold for the particular landslide type expressed in pShakeIntensity (Figure 8). Based on Keefer (2000) and Parise and Jibson (2000), landslide concentration should attenuate more rapidly with decreasing static susceptibility than decreasing shaking intensity. In further support of this constraint, Keefer (1984, Table 3$)$ observes that large numbers of landslides (1,000 to 10,000) are possible for moderate sized earthquakes (M 6). Lastly, the abundance table of Keefer (1984) (Table 28) was used in relating the seismic hazard rule blocks between the landslide types in CAMEL that have the same domain (or maximum possible concentration value). This was done by comparing the total number of each output fuzzy value as a proxy for abundance and ensuring the relative order of abundance is consistent with the observations of Keefer (1984). For example, the two rule blocks for disrupted soil slides, falls, and avalanches and for disrupted rock slides and rock falls are identical because these landslide types are approximately equally abundant. Conversely, the rule blocks for soil slumps and rock slumps are different, with more rules resulting in "very_high" concentration for soil slumps. This reflects the higher historical abundance of soil 
slumps and blocks slides over rock slumps and block slides.

\section{CAMEL Implementation and Use}

The previous section fully specified the fuzzy system that forms CAMEL. This section describes first how the fuzzy logic system design was implemented for computation of earthquake-induced landslide hazard. The components of the implemented fuzzy system, which can be integrated with Microsoft Excel ${ }^{\mathrm{TM}}$ or other COM (common object model)-compliant software programs, are presented. Second, the integration of the fuzzy logic system with ESRI ArcGIS ${ }^{\mathrm{TM}}$ for performing raster-based analysis is described. The setup, use and functionality of the ArcObjects ${ }^{\mathrm{TM}}$ tools are explained, followed by the logic and structure of the ArcObjects ${ }^{\mathrm{TM}}$ source code for CAMEL.

\subsection{Implementation of CAMEL}

The conceptual design of CAMEL, described in the above section, provides a blueprint that anyone can use to implement CAMEL using the programming language or fuzzy systems development tools of their choice. CAMEL is currently implemented using the fuzzy systems development software FuzzyTech ${ }^{\mathrm{TM}}$. With CAMEL implemented inside FuzzyTech, it is necessary to export the fuzzy system to facilitate use of the model outside of the development environment. The fuzzy system is exported as a single binary file ("CAMELddmmyy.ftr") that is used by the FuzzyTech Runtime DLL ("Ftrun32.dll”). (DLL stands for Dynamically Linked Libraries.) These and associated files are described in Table 19. The Runtime DLL can be integrated with Microsoft Excel ${ }^{\mathrm{TM}}, \mathrm{MATLAB}^{\mathrm{TM}}$ or other Microsoft Windows ${ }^{\mathrm{TM}}$ COMcompliant software. A specific example of integrating CAMEL with COM-compliant software is described below. (Integration with Microsoft Excel ${ }^{\mathrm{TM}}$ is considerably more straightforward than the below example, simply requiring a cell-function reference to "CAMELddmmyy.ftr" to assign each input variable and get each desired output.) 
Table 19. Description of FuzzyTech files for integrating CAMEL with COM-compliant software.

\section{FuzzyTech Runtime DLL Files}

Ftrun32.dll

The actual DLL file. Must be copied into “.../Windows/System32” folder and registered using regsvr32.exe.

Ftrun32.ini

Must be copied into ".../Windows" folder. A text file for modifying settings of the Runtime DLL. Instructions for use in "Ftrun32.hlp"

Ftrun32.hlp

Help file; typically installed in the same folder as "Ftrun32.dll". Double-click to open and read with Microsoft Help system.

Ftrun.bas

Visual Basic module file for use of "Ftrun32.dll" within Visual Basic or Visual Basic for Applications $^{\mathrm{TM}}$ (VBA) code. Wrappers for Visual $\mathrm{C}++^{\mathrm{TM}}$ and Java ${ }^{\mathrm{TM}}$ can also be created.

\section{CAMEL Files For FuzzyTech Runtime DLL}

CAMELddmmyy.ftr (e.g., CAMEL082805.ftr)

CAMEL fuzzy system (binary file) for use with "Ftrun32.dll." Any modifications to the fuzzy system require exporting a new .ftr file.

CAMELddmmyy.io

Text file describing input and output variables for accessing

"CAMELddmmyy.ftr."

CAMELddmmyy.cls

Visual Basic class file for use of "Ftrun32.dll" within Visual Basic or Visual Basic for Applications ${ }^{\mathrm{TM}}$ (VBA) code. Wrappers for Visual C $++^{\mathrm{TM}}$ and Java ${ }^{\mathrm{TM}}$ can also be created.

\subsection{Integration of CAMEL with ArcGIS ${ }^{\mathrm{TM}}$}

CAMEL was designed specifically to be a regional-scale model for predicting concentrations of all types of earthquake-induced landslides. Thus, at some level CAMEL must be integrated with GIS or other spatial technologies. Software integration and custom 


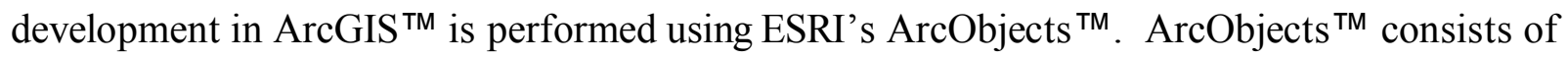
an object-oriented geographic data model and exposed software components, representing most, if not all, of ArcGIS ${ }^{\mathrm{TM}}$ functionality. ArcObjects ${ }^{\mathrm{TM}}$ is implemented using Microsoft's COM protocol. The advantage of this implementation is that any COM-compliant software development environment (e.g., Visual $\mathrm{C}++^{\mathrm{TM}}$ or Visual Basic ${ }^{\mathrm{TM}}$ ) can be used to customize ArcGIS $^{\mathrm{TM}}$ or add new functionality. Integration of CAMEL with ArcGIS ${ }^{\mathrm{TM}}$ was done using Visual Basic ${ }^{\mathrm{TM}}$.

At this stage of development, the main objective was to integrate most of CAMEL's functionality with ArcGIS ${ }^{\text {TM }}$ so that spatial data layers (input data) can be used to compute output layers for each landslide type. Developing a robust and user-friendly extension to ArcGIS was not an objective. Even so, CAMEL currently is relatively straightforward to setup and use within ArcGIS. After setup, CAMEL is accessed through an ArcGIS map document ("camelxx.mxd" with xx referring the compatible version of ArcGIS TM The map document contains several pre-loaded map layers for referencing input raster data, as well as a custom toolbar for running CAMEL.

\subsubsection{ArcGIS ${ }^{\mathrm{TM}}$ Setup and Use}

Setup of CAMEL for use with ArcGIS ${ }^{\mathrm{TM}}$ is simple. Copy the zip (archive) file "camelxx.zip" into a new folder, where "xx" is a two-digit number referring to the version of ArcGIS $^{\mathrm{TM}}$ the zipped files are compatible with (i.e., camel83.zip is compatible with version 8.3, while came190.zip is compatible with version 9.0). Double-click the zip file to open the archive and access its contents. The file "Ftrun32.dll" must be copied into the "C: $\mid$ Windows $\mid$ System32" folder on the computer, while "Ftrun32.ini" file must be copied into the "C:IWindows" folder. The "Ftrun32.dll" file must be registered by typing "regsvr32 ftrun32.dll" at the Windows command prompt. CAMEL then can be accessed by opening the map document (camelxx.mxd) in $\operatorname{ArcMap}^{\mathrm{TM}}$. Within the map document there is a custom toolbar (Figure 23) with three labeled toolbar buttons for running CAMEL: "Calc All Hazard”, "Calc Rock Hazard”, and "Calc Rock Hazard." The functions associated with each button are used in the same way and requires, for the most part, the same input layers. The difference is in the required layer for describing 
rock/soil properties, as well as the subsequent output layers (described below).

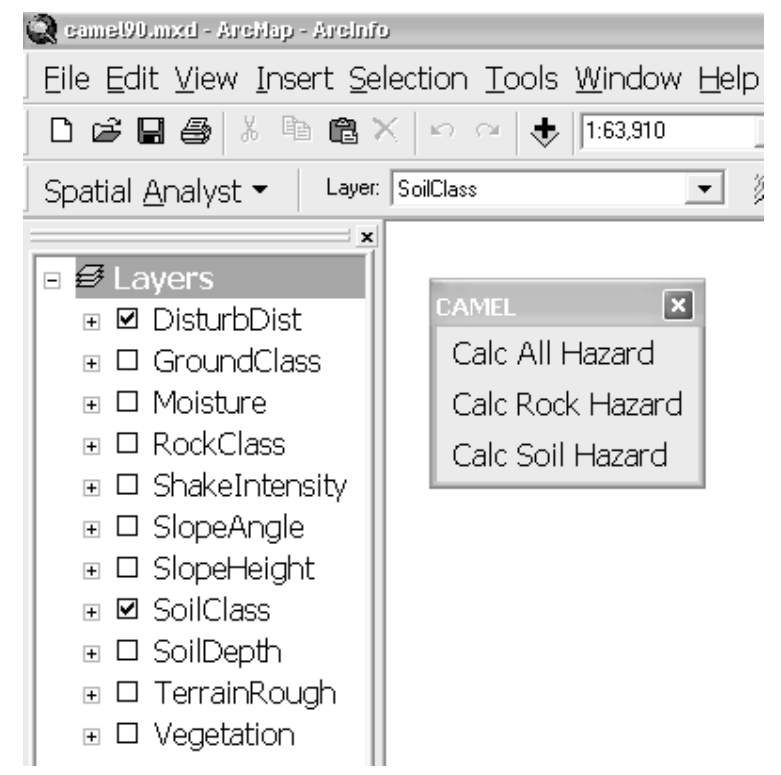

Figure 23. CAMEL implementation within ArcGISTM. Map layer names shown are required for CAMEL to find the respective input data.

Prior to running any of the functions, the input layers must be associated with the desired spatial data source for each input variable. The function looks in the ArcMap table of contents for specifically labeled raster map layers corresponding to each of CAMEL's input variables. The input layer labels adhere to a strict naming convention and capitalization in order for the function to find the layer and use it appropriately. The labeled input layers, reflecting the required label convention and capitalization, are listed in Figure 36. Any other layers added to the table of contents are ignored.

To associate the desired data with each respective input layer, right click on each layer in the table of contents and select "Data Source" to bring up the data source dialog (Figure 24). Click on the "Set Data Source" button in the dialog to find and assign the appropriate raster data set. This process must be done with each input layer, with the exception of "GroundClass", "RockClass" and "SoilClass." If the "Calc All Haz" function is to be used, the "GroundClass" layer must be specified. The "GroundClass" layer will then serve as an overall index of material properties for rock and soil; that is, the same properties will be used in assessing both rock and 
soil hazard. If "Calc Rock Hazard" is to be run, "RockClass" must be specified. If "Calc Soil Hazard" is to be run, "SoilClass" must be specified. While data can be missing for CAMEL (the fuzzy system) to run, currently the ArcGIS functions require that all layers be specified. So if no data is available, simply associate the particular layer with a raster of NO DATA. A 3x3-cell ArcGIS grid corresponding to each CAMEL input variable is provided in the camelxx.zip archive file in order to do a test setup and run of the model.

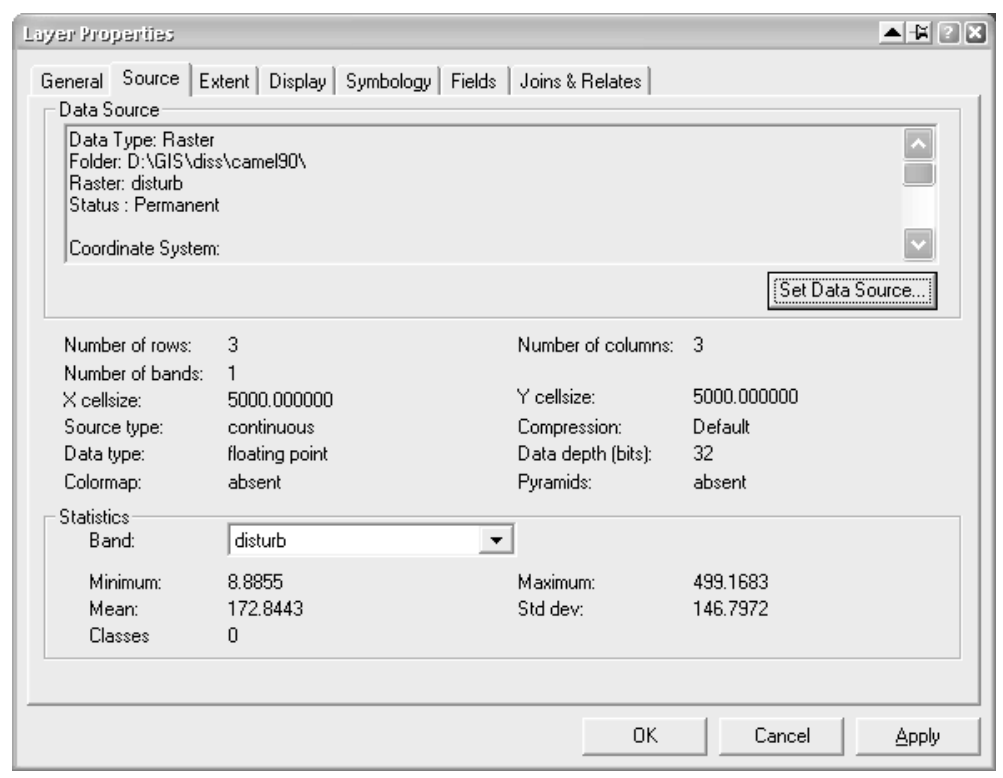

Figure 24. Data source dialog in ArcMap ${ }^{\mathrm{TM}}$ for assigning input raster data to appropriate input map layers.

Once the input data has been associated with the input layers, the respective CAMEL functions can be executed by clicking on the corresponding button in the CAMEL toolbar. The user is then presented with two input dialog boxes before hazard calculation begins (Figure 25). The first dialog box asks for a suffix text string that will be appended to each output raster file created. This is a convenience so that the user can run and keep track of outputs from several scenario (e.g., h_rockav1, h_rockav2; or h_rockav_wet, h_rockav_dry, etc.). The second dialog box asks for a minimum truth-value to be specified. The minimum truth-value reflects the lowest possibility value (from 0 to 1 ) that can be calculated for a particular landslide type and not be considered impossible (i.e., no hazard). 


\begin{tabular}{|c|c|c|c|}
\hline ATstilp & 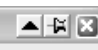 & Agrifip & $\Delta \leftarrow \bar{x}$ \\
\hline \multirow[t]{2}{*}{ Enter output filename suffix: } & $\mathrm{OK}$ & \multirow{2}{*}{$\begin{array}{l}\text { Enter minimum truth value (alpha } \\
\text { cut): }\end{array}$} & $\mathrm{OKK}$ \\
\hline & Cancel & & Cancel \\
\hline T & & \multicolumn{2}{|l|}{ I } \\
\hline
\end{tabular}

Figure 25. Input dialog boxes for running CAMEL functions.

After the second dialog box is completed, the respective function enumerates through all of the cells in the raster layer, passing the data values from each input cell to CAMEL (via the FuzzyTech $^{\mathrm{TM}}$ Runtime DLL). CAMEL then returns an array of landslide concentration values corresponding to either all six landslide types or just the three rock landslide types or just the three soil landslide types, depending on the particular function. New raster layers are created to store the respective array of output values from CAMEL. These layers are automatically added to the ArcMap table of contents and appropriately labeled (Figure 26). Currently, in order to run another scenario, it is necessary to change the names of the previously created output layers (e.g., from "Soil Slump Hazard” to "Soil Slump Hazard Dry"). 


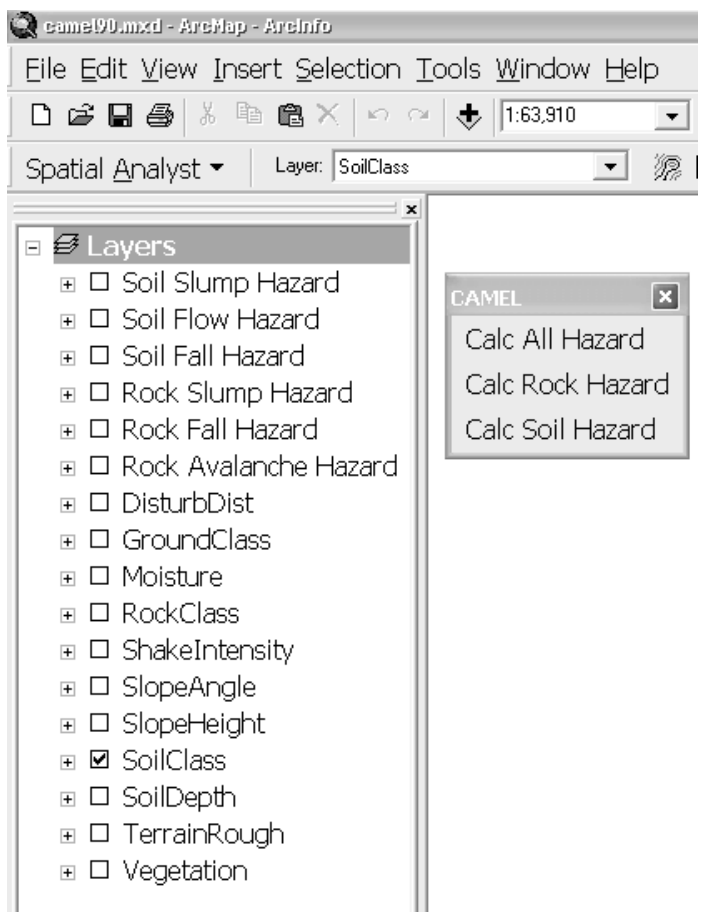

Figure 26. Example of output layers created after running "Calc All Hazard."

\section{References}

Berkan, R. and Trubatch, S. (1997) Fuzzy Systems Design Principles: Building Fuzzy IF-THEN Rule Bases. New York: IEEE Press.

Bommer, J. and Rodriguez, C. (2002) Earthquake-induced landslides in Central America. Engineering Geology, 63, 189-220.

Combs, W.E. and Andrews, J.E. (1998) Combinatorial rule explosion eliminated by a fuzzy rule configuration. IEEE Transactions on Fuzzy Systems, 6, 1-11.

Cruden, D. and Varnes, D. (1996) Landslide types and processes. in A. Turner and R. Schuster (Ed.), Landslides: Investigations and Mitigation (pp. 36-75). Washington, D.C.: Transportation Research Board.

Guillaume, S. (2001) Designing fuzzy inference systems from data: An interpretability-oriented review. IEEE Transactions on Fuzzy Systems, 9, pp 426-443.

Hancox, G., Perrin, N. and Dellow, G. (2002) Recent studies of historical earthquake-induced landsliding, ground damange, and MM intensity in New Zealand. Bulletin of the New Zealand Society for Earthquake Engineering, 35, 59-95. 
Hansen, A. and Franks, C. (1991) Characterisation and mapping of earthquake triggered landslides for seismic zonation. Proceedings of the Fourth International Conference on Seismic Zonation (pp. 149-195). Palo Alto, CA.

Jibson, R., Harp, E., and Michael, J. (1998) A method for producing digital probabilistic seismic landslide hazard maps. U.S. Geological Survey Open-File Report 98-113, 17, 2 pl.

Keefer, D.K. (1984) Landslides caused by earthquakes. Bulletin of the Geological Society of America, 95, 406-421.

Keefer, D.K. (1993) The susceptibility of rock slopes to earthquake-induced failure. Association of Engineering Geologists Bulletin, 30, 353--361.

Keefer, D.K. (Ed.) (1998) The Loma Prieta, California, Earthquake of October 17, 1989 -Landslides. U.S. Geological Survey Professional Paper 1551-C.

Keefer, D.K. (2000) Statistical analysis of an earthquake-induced landslide distribution - The 1989 Loma Prieta, California event. Engineering Geology, 58, 231-249.

Lazzerini, B. and Marcelloni, F.. (2000) Reducing computation overhead in MISO fuzzy systems. Fuzzy Sets and Systems, 113, 485-496.

Liao, C., Lee C.T., Liao, and Liao, H. (2002) Statistical analysis of factors affecting landslides triggered by the 1999 Chi-Chi, Taiwan Earthquake. American Geophysical Union Meeting. San Francisco.

Parise, M. and Jibson, R. (2000) A seismic landslide susceptibility rating of geologic units based on analysis of characteristics of landslides triggered by the 17 January, 1994 Northridge, California earthquake. Engineering Geology, 58, 251-270.

Rodriguez, C., Bommer, J. and Chandler, R. (1999) Earthquake-induced landslides: 1980-1997. Soil Dynamics and Earthquake Engineering, 18, 325-346.

Wald, D.Q.V., Heaton, T., Kanamori, H., Scrivner, C. and Worden, C. (1999) TriNet "ShakeMaps": Rapid generation of instrumental ground motion and intensity maps for earthquakes in Southern California. Earthquake Spectra, 15, 537-556.

Zadeh, L. (1965) Fuzzy sets. Information and Control, 8, 338-353. 


\section{Appendix A: Complete Specification of Hazard Module Rule- Blocks for CAMEL}

This appendix presents all of the rule-blocks and corresponding rules that comprise the hazard module of CAMEL. The three rule-blocks - static susceptibility, modifiers, and seismic hazard - are presented in turn for each of the six landslide types considered by CAMEL. All rule-blocks use PROD operators if there is more than one input and BSUM aggregation.

\section{A.1 Rock Avalanche}

Table A1. Static susceptibility rule block for rock avalanches.

\begin{tabular}{|l|l|l|l|l|}
\hline \multicolumn{3}{|c|}{ IF } & \multicolumn{2}{c|}{ THEN } \\
\hline \multicolumn{1}{|c|}{ hGroundClass } & LandslideType & hSlopeAngle & DoS & RAvStaticInt \\
\hline very_good & rock_avalanche & & 0.75 & very_low \\
\hline good & rock_avalanche & & 0.75 & low \\
\hline moderate & rock_avalanche & & 0.75 & medium \\
\hline poor & rock_avalanche & & 0.75 & high \\
\hline very_poor & rock_avalanche & & 0.75 & very_high \\
\hline & rock_avalanche & medium & 0.25 & very_low \\
\hline & rock_avalanche & medium_steep & 0.25 & low \\
\hline & rock_avalanche & steep_ & 0.25 & medium \\
\hline & rock_avalanche & very_steep & 0.25 & high \\
\hline & rock_avalanche & extremely_steep & 0.25 & very_high \\
\hline & rock_avalanche & nearl_vertical & 0.40 & very_high \\
\hline
\end{tabular}


Table A2. Modifiers rule block for rock avalanches.

\begin{tabular}{|l|l|l|l|}
\hline \multicolumn{2}{|c|}{ IF } & \multicolumn{2}{c|}{ THEN } \\
\hline hDisturbanceDist & RAvStaticInt & DoS & RAvStatic \\
\hline & very_low & 1.00 & very_low \\
\hline & low & 1.00 & low \\
\hline & medium & 1.00 & medium \\
\hline & high__igh & 1.00 & high \\
\hline & very_high & 1.00 & very_high \\
\hline close & very_low & 0.50 & low \\
\hline close & low & 0.50 & medium \\
\hline close & medium & 0.50 & high \\
\hline close & high & 0.50 & very_high \\
\hline close & very_high & 0.50 & very_high \\
\hline
\end{tabular}

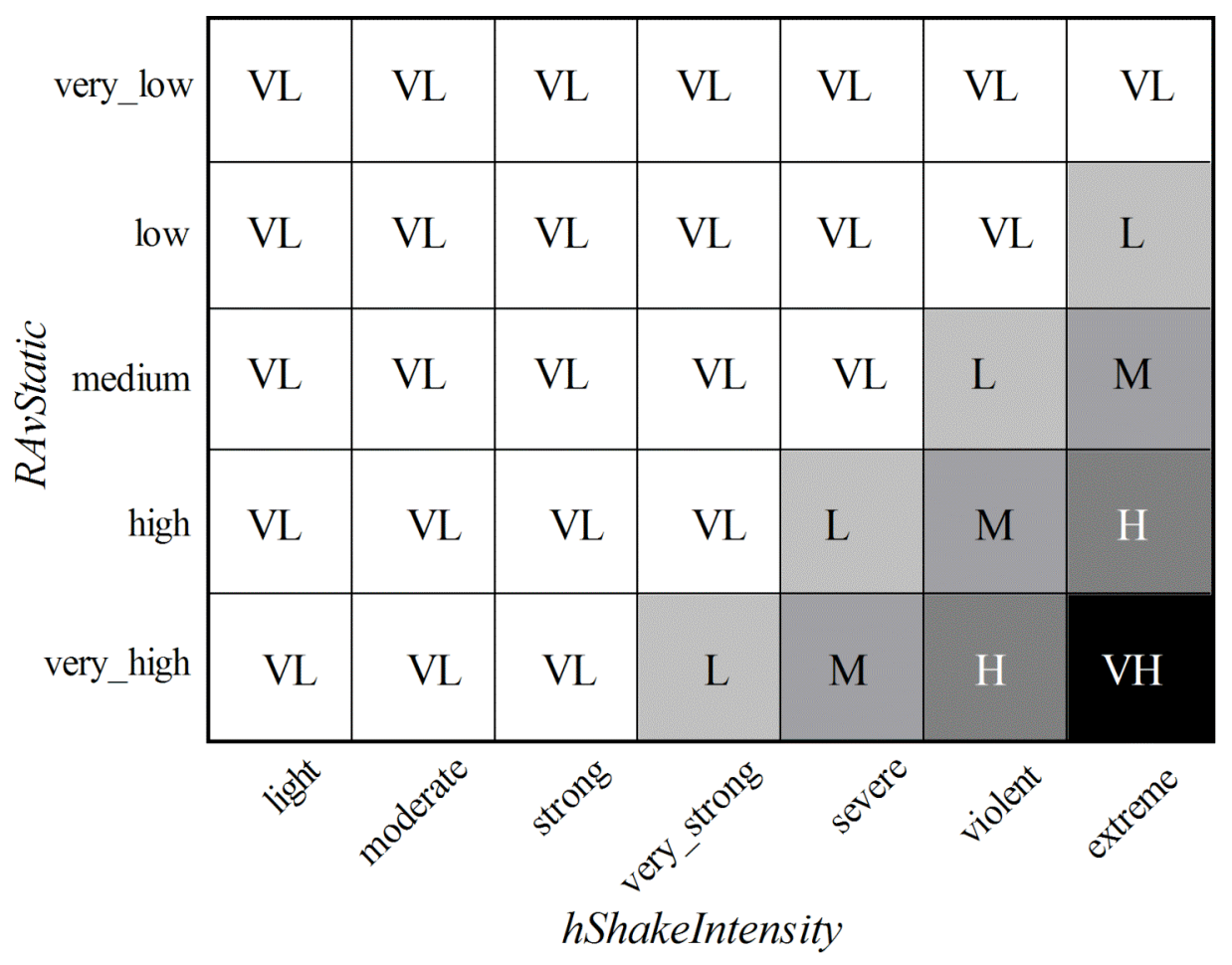

Figure A1. Seismic hazard rule block for rock avalanches. $V L=$ very_low, $L=l o w, M=$ medium, $\mathrm{H}=$ high, $\mathrm{VH}=$ very_high. 


\section{A.2 Rock Fall and Disrupted Rock Slides}

Table A3. Static susceptibility rule block for disrupted rock falls and slides.

\begin{tabular}{|l|l|l|l|l|}
\hline \multicolumn{3}{|c|}{ IF } & \multicolumn{2}{c|}{ THEN } \\
\hline hGroundClass & LandslideType & hSlopeAngle & DoS & RFallStaticInt \\
\hline very_good & rock_fall & & 0.80 & very_low \\
\hline good & rock_fall & & 0.80 & low \\
\hline moderate & rock_fall & & 0.80 & medium \\
\hline poor & rock_fall & & 0.80 & high \\
\hline very_poor & rock_fall & & 0.80 & very_high \\
\hline & rock_fall & medium_steep & 0.20 & very_low \\
\hline & rock_fall & steep & 0.20 & low \\
\hline & rock_fall & very_steep & 0.20 & medium \\
\hline & rock_fall & extremely_steep & 0.20 & high \\
\hline & rock_fall & nearl_vertical & 0.20 & very_high \\
\hline
\end{tabular}

Table A4. Modifiers rule block for disrupted rock falls and slides.

\begin{tabular}{|l|l|l|l|l|l|}
\hline \multicolumn{2}{|c|}{ IF } & \multicolumn{2}{c|}{ THEN } \\
\hline $\begin{array}{c}\text { hDisturbance } \\
\text { Dist }\end{array}$ & hMoisture & RFallStaticInt & hVegetation & DoS & RFallStatic \\
\hline & & very_low & dense & 1.00 & very_low \\
\hline & & low & dense & 1.00 & very_low \\
\hline & & very_low & & 1.00 & very_low \\
\hline & & medium & dense & 0.34 & low \\
\hline & vvery_low & very_low & & 0.34 & low \\
\hline close & & low & & 1.00 & low \\
\hline & & very_low & & 0.34 & low \\
\hline & & high & dense & 0.34 & medium \\
\hline & $\neg$ very_low & low & & 0.34 & medium \\
\hline close & & medium & & 1.00 & medium \\
\hline & & low & & 0.34 & medium \\
\hline & & very_high & dense & 0.34 & high \\
\hline & $\neg$ very_low & medium & & 0.34 & high \\
\hline close & & high & & 1.00 & high \\
\hline & & medium & & 0.34 & high \\
\hline & $\neg$ very_low & very_high & & 0.34 & very_high \\
\hline & $\neg$ very_low & high & & 0.34 & very_high \\
\hline close & & very_high & & 1.00 & very_high \\
\hline close & & high & & 0.34 & very_high \\
\hline & & very_high & & 0.34 & very_high \\
\hline
\end{tabular}




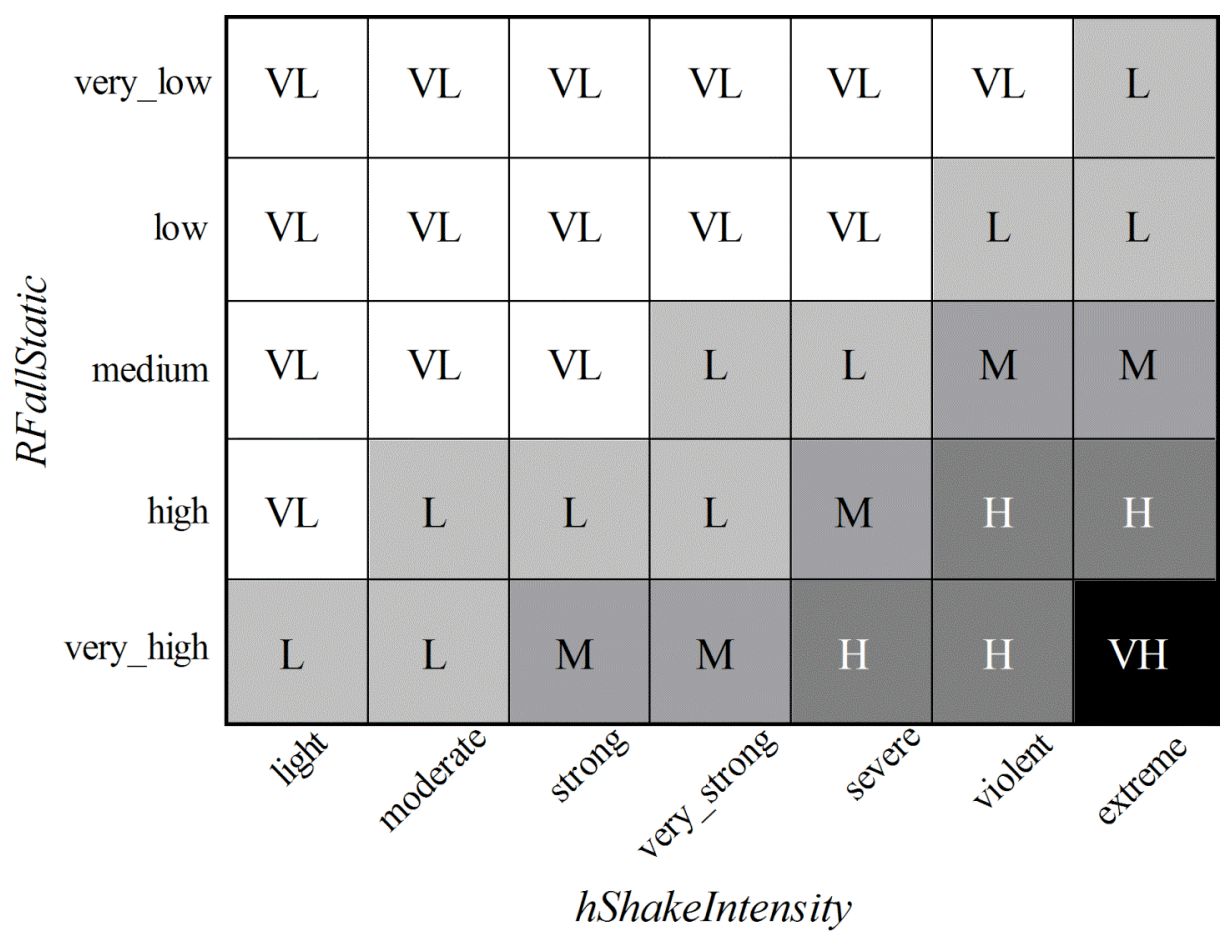


Figure A2. Seismic hazard rule block for disrupted rock slides and falls. VL = very_low, $L$ = low, $\mathrm{M}=$ medium, $\mathrm{H}=$ high, $\mathrm{VH}=$ very_high.

\section{A.3 Rock Slumps and Rock Block Slides}

Table A5. Static susceptibility rule block for rock slumps and block slides.

\begin{tabular}{|c|c|c|c|c|c|}
\hline \multicolumn{4}{|c|}{ IF } & \multicolumn{2}{|c|}{ THEN } \\
\hline $\begin{array}{c}\text { hGround } \\
\text { Class } \\
\end{array}$ & $\begin{array}{c}\text { Landslide } \\
\text { Type }\end{array}$ & hMoisture & hSlopeAngle & DoS & $\begin{array}{l}\text { RSlump } \\
\text { StaticInt } \\
\end{array}$ \\
\hline very_good & rock_slump & & & 0.40 & very_low \\
\hline good & rock_slump & & & 0.40 & low \\
\hline moderate & rock_slump & & & 0.40 & medium \\
\hline poor & rock_slump & & & 0.40 & high \\
\hline very_poor & rock_slump & & & 0.40 & very_high \\
\hline & rock_slump & very_low & & 0.40 & very_low \\
\hline & rock slump & low & & 0.40 & low \\
\hline & rock_slump & medium & & 0.40 & medium \\
\hline & rock slump & high & & 0.40 & high \\
\hline & rock_slump & very_high & & 0.40 & very_high \\
\hline & rock_slump & & gradual & 0.20 & very_low \\
\hline & rock_slump & & medium_gradual & 0.20 & low \\
\hline & rock_slump & & medium & 0.20 & medium \\
\hline & rock_slump & & medium_steep & 0.20 & high \\
\hline & rock_slump & & steep & 0.20 & very_high \\
\hline & rock_slump & & very_steep & 0.30 & very_high \\
\hline & rock slump & & extremely steep & 0.40 & very high \\
\hline & rock_slump & & nearl_vertical & 0.50 & very_high \\
\hline
\end{tabular}


Table A6. Modifiers rule block for rock slumps and block slides.

\begin{tabular}{|l|l|l|l|l|}
\hline \multicolumn{3}{|c|}{ IF } & \multicolumn{2}{c|}{ THEN } \\
\hline hDisturbanceDist & RSlumpStaticInt & hVegetation & DoS & RSlumpStatic \\
\hline & very_low & & 1.00 & very_low \\
\hline & very_low & dense & 1.00 & very_low \\
\hline & low & dense & 1.00 & very_low \\
\hline & low & & 1.00 & low \\
\hline & medium & dense & 0.50 & low \\
\hline close & very_low & & 0.50 & low \\
\hline & medium & & 1.00 & medium \\
\hline & high & dense & 0.50 & medium \\
\hline close & low & & 0.50 & medium \\
\hline & high & & 1.00 & high \\
\hline close & very_high & dense & 0.50 & high \\
\hline & medium & & 0.50 & high \\
\hline close & very_high & & 1.00 & very_high \\
\hline close & high & & 1.00 & very_high \\
\hline
\end{tabular}

\begin{tabular}{|c|c|c|c|c|c|c|c|}
\hline very_low & VL & VL & VL & VL & VL & VL & VL \\
\hline low & VL & VL & VL & VL & VL & $\mathrm{L}$ & $\mathrm{L}$ \\
\hline $\begin{array}{l}\overrightarrow{\widetilde{\Xi}} \text { medium } \\
\text { इ }\end{array}$ & VL & VL & VL & VL & L & M & M \\
\hline$\approx \quad$ high & VL & VL & VL & $\mathrm{L}$ & M & $\mathrm{H}$ & H \\
\hline very_high & VL & VL & $\mathrm{L}$ & M & $\mathrm{H}$ & $\mathrm{H}$ & VH \\
\hline
\end{tabular}

Figure A3. Seismic hazard rule block for rock slumps and block slides. $V L=$ very_low, $L=$ low, $\mathrm{M}=$ medium, $\mathrm{H}=$ high, $\mathrm{VH}=$ very_high. 


\section{A.4 Disrupted Soil Slides, Soil Falls, and Soil Avalanches}

Table A7. Static susceptibility rule block for soil falls, slides, and avalanches.

\begin{tabular}{|l|l|l|l|l|}
\hline \multicolumn{2}{|c|}{ IF } & \multicolumn{2}{c|}{ THEN } \\
\hline hGroundClass & LandslideType & hSlopeAngle & DoS & SFallStaticInt \\
\hline very_good & soil_fall & & 0.75 & very_low \\
\hline good & soil_fall & & 0.75 & low \\
\hline moderate & soil_fall & & 0.75 & medium \\
\hline poor & soil_fall & & 0.75 & high \\
\hline very_poor & soil_fall & & 0.75 & very_high \\
\hline & soil_fall & gradual_ & 0.25 & very_low \\
\hline & soil_fall & medium_gradual & 0.25 & low \\
\hline & soil_fall & medium_ & 0.25 & low \\
\hline & soil_fall & medium_steep & 0.25 & medium \\
\hline & soil_fall & steep & 0.25 & medium \\
\hline & soil_fall & very_steep & 0.25 & high \\
\hline & soil_fall & extremely_steep & 0.25 & high \\
\hline & soil_fall & nearl_vertical & 0.25 & very_high \\
\hline
\end{tabular}


Table A8. Modifiers rule block for disrupted soil falls, slides and avalanches.

\begin{tabular}{|c|c|c|c|c|c|}
\hline \multicolumn{4}{|c|}{ IF } & \multicolumn{2}{|c|}{ THEN } \\
\hline DisturbanceDist & Moisture & $\begin{array}{l}\text { SFallStaticI } \\
\text { nt }\end{array}$ & Vegetation & DoS & SFallStatic \\
\hline & & very_low & dense & 1.00 & very_low \\
\hline & & low & dense & 1.00 & very_low \\
\hline & & very low & & 1.00 & very_low \\
\hline & & medium & dense & 0.34 & low \\
\hline close & & very_low & & 0.34 & low \\
\hline & ᄀvery low & very low & & 0.34 & low \\
\hline & & low & & 1.00 & low \\
\hline & & high & dense & 0.34 & medium \\
\hline close & & low & & 0.34 & medium \\
\hline & $\neg$ very_low & low & & 0.34 & medium \\
\hline & & medium & & 1.00 & medium \\
\hline & & very_high & dense & 0.34 & high \\
\hline close & & medium & & 0.34 & high \\
\hline & $\neg$ very low & medium & & 0.34 & high \\
\hline & & high & & 1.00 & high \\
\hline close & & high & & 0.50 & very_high \\
\hline close & & very high & & 0.50 & very high \\
\hline & $\neg$ very_low & high & & 0.50 & very_high \\
\hline & $\neg$ very_low & very_high & & 0.50 & very_high \\
\hline & & very_high & & 1.00 & very high \\
\hline
\end{tabular}




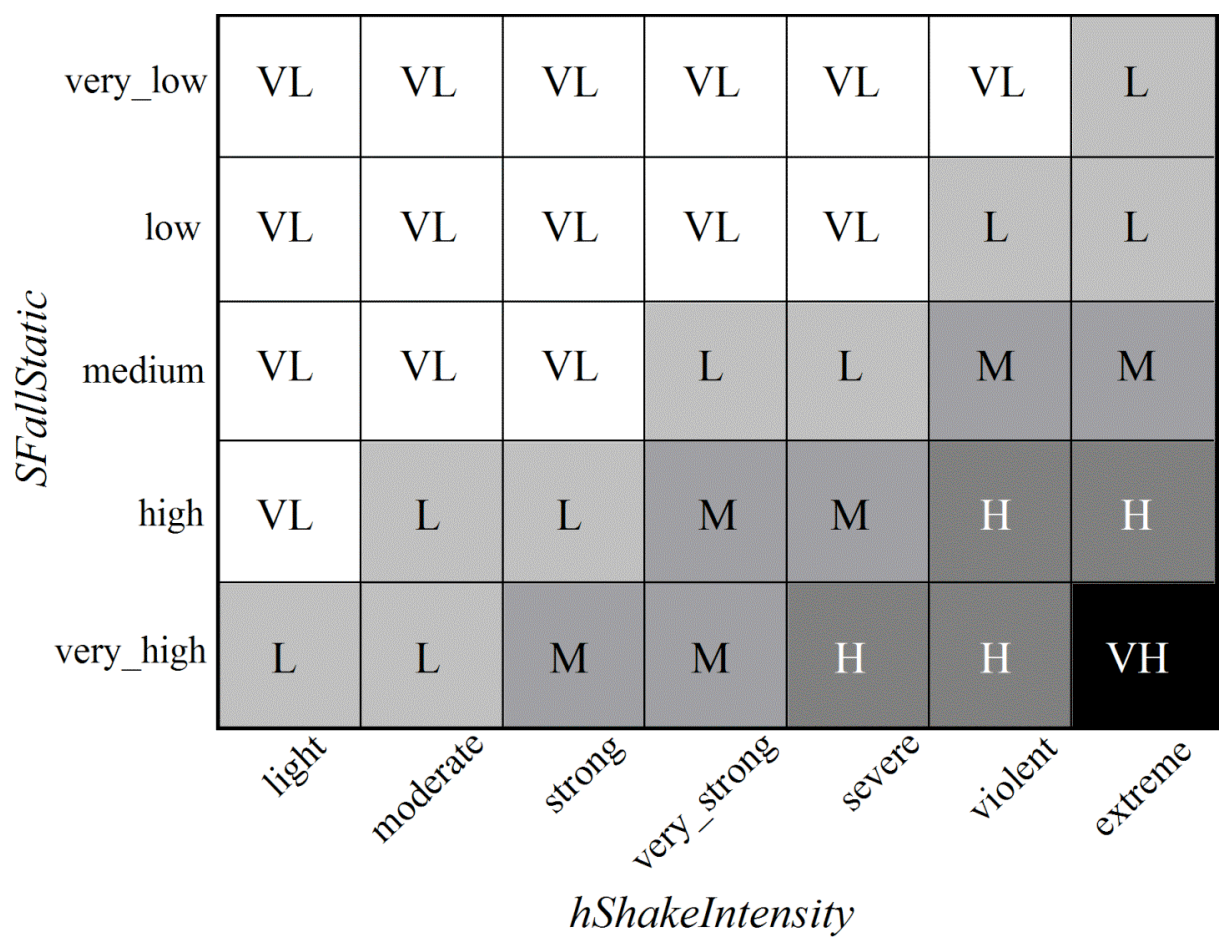

Figure A4. Seismic hazard rule block for disrupted soil slides and falls. VL=very_low, L = low, $\mathbf{M}=$ medium, $\mathrm{H}=$ high, $\mathrm{VH}=$ very_high . 


\section{A.5 Soil Slumps and Soil Block Slides}

Table A9. Static susceptibility rule block for soil slumps and block slides.

\begin{tabular}{|c|c|c|c|c|c|}
\hline \multicolumn{4}{|c|}{ IF } & \multicolumn{2}{|c|}{ THEN } \\
\hline $\begin{array}{c}\text { Ground } \\
\text { Class } \\
\end{array}$ & $\begin{array}{c}\text { Landslide } \\
\text { Type } \\
\end{array}$ & Moisture & SlopeAngle & DoS & $\begin{array}{c}\text { SSlump } \\
\text { StaticInt } \\
\end{array}$ \\
\hline & soil_slump & & very gradual & 0.20 & very low \\
\hline & soil_slump & & gradual & 0.20 & very low \\
\hline & soil_slump & & medium_gradual & 0.20 & low \\
\hline & soil_slump & & medium & 0.20 & medium \\
\hline & soil_slump & & medium_steep & 0.20 & high \\
\hline & soil_slump & & steep & 0.20 & very_high \\
\hline & soil_slump & & very_steep & 0.30 & very_high \\
\hline & soil_slump & & extremely_steep & 0.40 & very high \\
\hline & soil_slump & & nearl_vertical & 0.50 & very_high \\
\hline very_good & soil_slump & & & 0.40 & very low \\
\hline good & soil_slump & & & 0.40 & low \\
\hline moderate & soil_slump & & & 0.40 & medium \\
\hline poor & soil_slump & & & 0.40 & high \\
\hline very_poor & soil_slump & & & 0.40 & very_high \\
\hline & soil_slump & very_low & & 0.40 & very_low \\
\hline & soil_slump & low & & 0.40 & low \\
\hline & soil_slump & medium & & 0.40 & medium \\
\hline & soil_slump & high & & 0.40 & high \\
\hline & soil_slump & very_high & & 0.40 & very high \\
\hline
\end{tabular}

Table A10. Modifiers rule block for soil slumps and block slides. 


\begin{tabular}{|l|l|l|l|l|}
\hline \multicolumn{3}{|c|}{ IF } & \multicolumn{2}{c|}{ THEN } \\
\hline DisturbanceDist & SSlumpStaticInt & Vegetation & DoS & SSlumpStatic \\
\hline & very_low & dense & 1.00 & very_low \\
\hline & low & dense & 1.00 & very_low \\
\hline & very_low & & 1.00 & very_low \\
\hline & medium & dense & 0.50 & low \\
\hline & low & & 1.00 & low \\
\hline close & very_low & & 0.50 & low \\
\hline & high & dense & 0.50 & medium \\
\hline close & medium & & 1.00 & medium \\
\hline & low & & 0.50 & medium \\
\hline & very_high & dense & 0.50 & high \\
\hline close & high & & 1.00 & high \\
\hline & medium & & 0.50 & high \\
\hline close & very_high & & 1.00 & very_high \\
\hline close & high & & 1.00 & very_high \\
\hline
\end{tabular}

\begin{tabular}{|c|c|c|c|c|c|c|c|}
\hline very_low & VL & VL & VL & VL & VL & VL & L \\
\hline low & VL & VL & VL & VL & L & $\mathrm{L}$ & M \\
\hline § & VL & VL & VL & $\mathrm{L}$ & M & M & H \\
\hline ऽ high & VL & VL & L & M & H & $\mathrm{H}$ & VH \\
\hline very_high & VL & $\mathrm{L}$ & M & H & $\mathrm{H}$ & VH & VH \\
\hline
\end{tabular}

Figure A5. Seismic hazard rule block for soil slumps and block slides. VL $=$ very_low, $L=$ low, $\mathrm{M}=$ medium, $\mathrm{H}=$ high, $\mathrm{VH}=$ very_high.

\section{A.6 Rapid Soil Flows}

Table A11. Static susceptibility rule block for rapid soil flows. 


\begin{tabular}{|l|l|l|l|l|}
\hline \multicolumn{3}{|c|}{ IF } & \multicolumn{2}{c|}{ THEN } \\
\hline GroundClass & LandslideType & SlopeAngle & DoS & SFlowStaticInt \\
\hline very_good & soil_flow & & 0.75 & very_low \\
\hline good & soil_flow & & 0.75 & low \\
\hline moderate & soil_flow & & 0.75 & medium \\
\hline poor & soil_flow & & 0.75 & high \\
\hline very_poor & soil_flow & & 0.75 & very_high \\
\hline & soil_flow & very_gradual & 0.25 & very_low \\
\hline & soil_flow & gradual & 0.25 & very_low \\
\hline & soil_flow & medium_gradual & 0.25 & low \\
\hline & soil_flow & medium & 0.25 & low \\
\hline & soil_flow & medium_steep & 0.25 & medium \\
\hline & soil_flow & steep & 0.25 & medium \\
\hline & soil_flow & very_steep & 0.25 & high \\
\hline & soil_flow & extremely_steep & 0.25 & high \\
\hline & soil_flow & nearl_vertical & 0.25 & very_high \\
\hline
\end{tabular}

Table A12. Modifiers rule block for rapid soil flows.

\begin{tabular}{|c|c|c|c|c|c|}
\hline \multicolumn{4}{|c|}{ IF } & \multicolumn{2}{|c|}{ THEN } \\
\hline $\begin{array}{c}\text { Disturbance } \\
\text { Dist }\end{array}$ & Moisture & $\begin{array}{c}\text { SFlow } \\
\text { StaticInt }\end{array}$ & Vegetation & DoS & $\begin{array}{c}\text { SFlow } \\
\text { Static }\end{array}$ \\
\hline & & very low & dense & 1.00 & very low \\
\hline & & low & dense & 1.00 & very low \\
\hline & & very_low & & 1.00 & very_low \\
\hline & very_high & very_low & & 0.34 & low \\
\hline & & medium & dense & 0.34 & low \\
\hline & & low & & 1.00 & low \\
\hline close & & very low & & 0.34 & low \\
\hline & very high & low & & 0.34 & medium \\
\hline & & high & dense & 0.34 & medium \\
\hline & & medium & & 1.00 & medium \\
\hline close & & low & & 0.34 & medium \\
\hline & very_high & medium & & 0.34 & high \\
\hline & & very_high & dense & 0.34 & high \\
\hline & & high & & 1.00 & high \\
\hline close & & medium & & 0.34 & high \\
\hline & very_high & high & & 0.50 & very high \\
\hline & very high & very high & & 0.50 & very high \\
\hline & & very high & & 1.00 & very high \\
\hline close & & high & & 0.50 & very high \\
\hline close & & very_high & & 0.50 & very_high \\
\hline
\end{tabular}




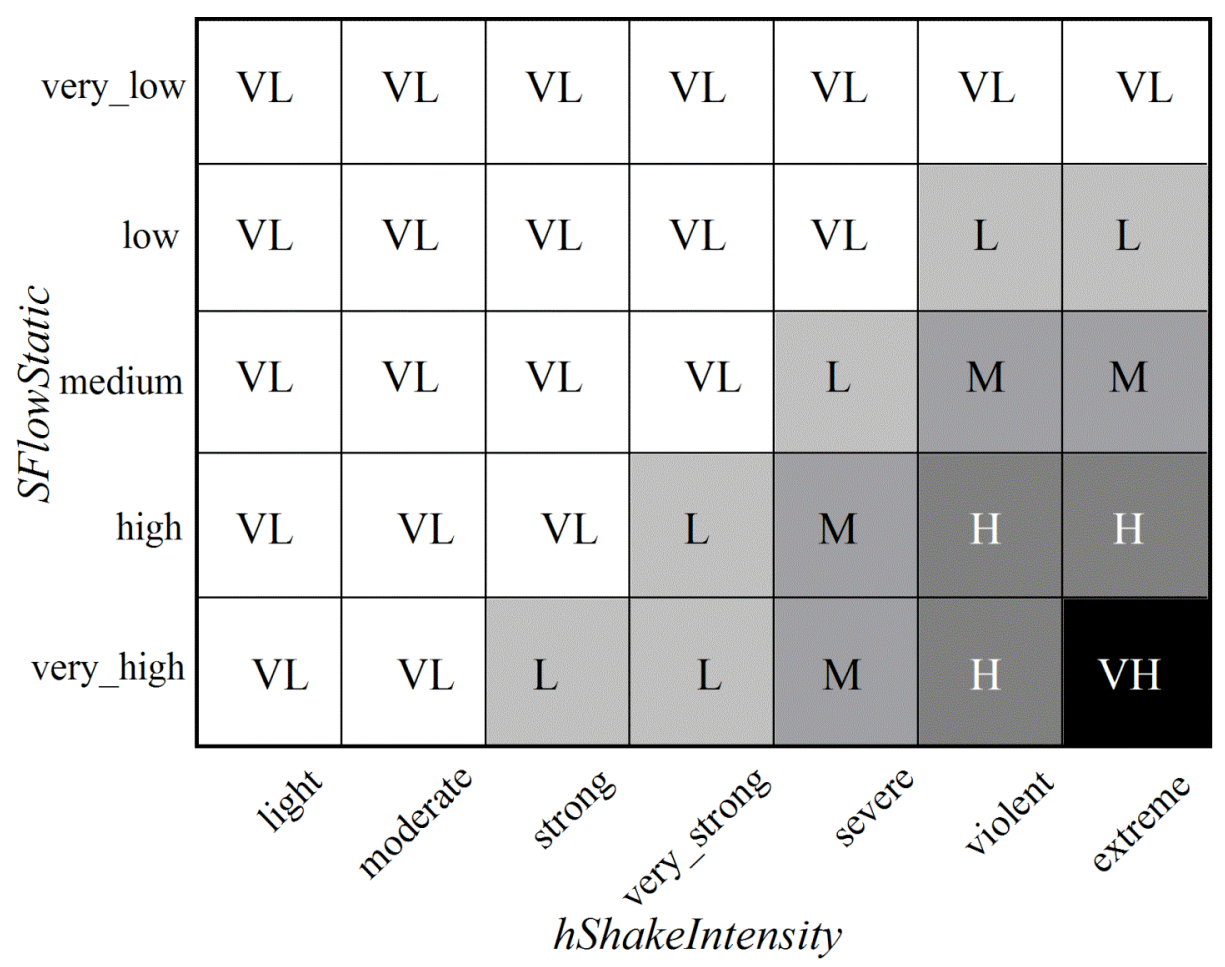

Figure A6. Seismic hazard rule block for rapid soil flows. $V L=$ very_low, $L=l o w, M=$ medium, $H=$ high, $V H=$ very_high. 